\title{
How to implement user-defined fiber-reinforced hyperelastic materials in finite element software
}

\author{
Heleen Fehervary ${ }^{1 \mathrm{a}}$, Lauranne Mae ${ }^{1 \mathrm{l}}{ }^{\mathrm{a}}$, Julie Vastmans ${ }^{\mathrm{a}}$, Gertjan \\ Kloosterman ${ }^{\mathrm{b}}$, Nele Famaey ${ }^{\mathrm{a},}$ \\ ${ }^{a}$ Biomechanics Section, Mechanical Engineering Department, KU Leuven, Leuven, Belgium \\ ${ }^{b}$ Mechanical Engineering, Institute for Engineering and Design, University of Applied \\ Sciences Utrecht, Utrecht, The Netherlands
}

\begin{abstract}
Finite element modeling is often used in biomechanical engineering to evaluate medical devices, treatments and diagnostic tools. Using an adequate material model that describes the mechanical behavior of biological tissues is essential for a reliable outcome of the simulation. Pre-programmed material models for biological tissues are available in many finite element software packages. However, since these pre-programmed models are presented to the user as a black box, without the possibility to modify the material description, many researchers turn to implementing their own material formulations. This is a complex undertaking, requiring extensive knowledge while documentation is limited.

This paper provides a detailed description, at the level of the biomedical engineer, of the implementation of a nonlinear hyperelastic material model using user subroutines in Abaqus ${ }^{\circledR}$, in casu UANISOHYPER_INV and UMAT. The Gasser-Ogden-Holzapfel material model is used as an example, resulting in four implementation variations: the built-in implementation, a UANISOHYPER_INV formulation, a UMAT with analytical tangent stiffness formulation and a UMAT with numerical tangent stiffness formulation. In addition, three different element formulations are used: a continuum compressible, a continuum incompressible and a plane stress incompressible. All cases are thoroughly verified by applying a series of deformations on a single cube element and by simulating an
\end{abstract}

\footnotetext{
${ }^{1}$ These authors contributed equally to this work.
} 
extension-inflation experiment with non-homogeneous deformations and multiple elements. In these test cases, stresses, displacements, reaction forces, the required number of iterations and the total $\mathrm{CPU}$ time were compared.

The results show that the four implementation variations are very similar, with total relative errors between $10^{-3}$ and $10^{-15}$, number of iterations that varied by maximum one iteration, and a comparable CPU time. In addition to this detailed overview, the user subroutines are added as supplementary material to this tutorial, which can be used as the ideal starting point for biomechanical engineers to implement their own material models at different levels of complexity.

Key words: finite element modeling, user defined material, constitutive modeling, anisotropy

\section{Introduction}

In biomedical engineering, finite element (FE) modeling is often used for the evaluation of medical devices, treatments and diagnostic tools. An adequate material model that describes the mechanical behavior of soft biological tissues

5 is essential for a reliable outcome of the simulation. Soft biological tissues often exhibit complex material properties such as a nonlinear and anisotropic stress-strain response, viscoelasticity, active behavior (e.g. caused by smooth muscle cells), poroelasticity (e.g. cartilage) and the ability to grow and remodel. Different constitutive models have been developed to address (a subset of) these material properties. In most commercially available FE software packages, some pre-programmed material models for soft biological tissues are available. For nonlinear, anisotropic materials, the Gasser-Ogden-Holzapfel (GOH) material, that was originally developed to model arterial tissue [1, is often provided.

The disadvantage of these pre-programmed models is that they are presented to the user as a black box. Although changing material parameters is very easy, modifications such as adding fiber families or including inelastic behavior (e.g. growth, viscoelasticity or poroelasticity), is not possible in these 
pre-programmed models. Therefore, many researchers turn to implementing their own material formulations through user subroutines. This is a complex undertaking, requiring rather extensive knowledge of continuum mechanics and tensor algebra. In addition, even with available documentation such as FE software manuals, books and research papers, it is not straightforward to acquire this knowledge. Often these documents only provide expressions, without any derivations, lack details and background information, making it difficult to extrapolate to other material models and can consequently lead to unconscious errors.

The goal of this tutorial is to provide a theoretical overview, at the level of the biomechanical engineer, and a step-by-step explanation on how to implement a nonlinear hyperelastic, fiber-reinforced material formulation into the commercial so software program Abaqus ${ }^{\circledR}$ using a user material subroutine (UANISOHYPER_INV or UMAT). We choose the GOH material as an example, since it allows us to verify our methodology against the pre-programmed version.

In this manuscript, the materials and methods section 2 describes the chosen example material model, different possible implementations of this model, as well as the test cases used to verify these implementations. Both solid and shell elements are considered. In the results section 3 we verify and compare the different implementations based on accuracy of the stresses, forces, displacements and number of iterations. Finally, these results are discussed and a conclusion is formulated.

\section{2. Materials and Methods}

This section starts with a description of the coordinate systems that can be considered in a FE simulation, followed by the description of the GOH material model [1] which is used as an example material throughout the paper. Next, different approaches to implement this material model in $\mathrm{FE}$ software via user

45 subroutines are explained. Finally, the test cases used to compare and verify the different implementations are described. 


\subsection{Coordinate systems}

In general, three coordinate systems can be considered in a FE simulation (see figure 1):

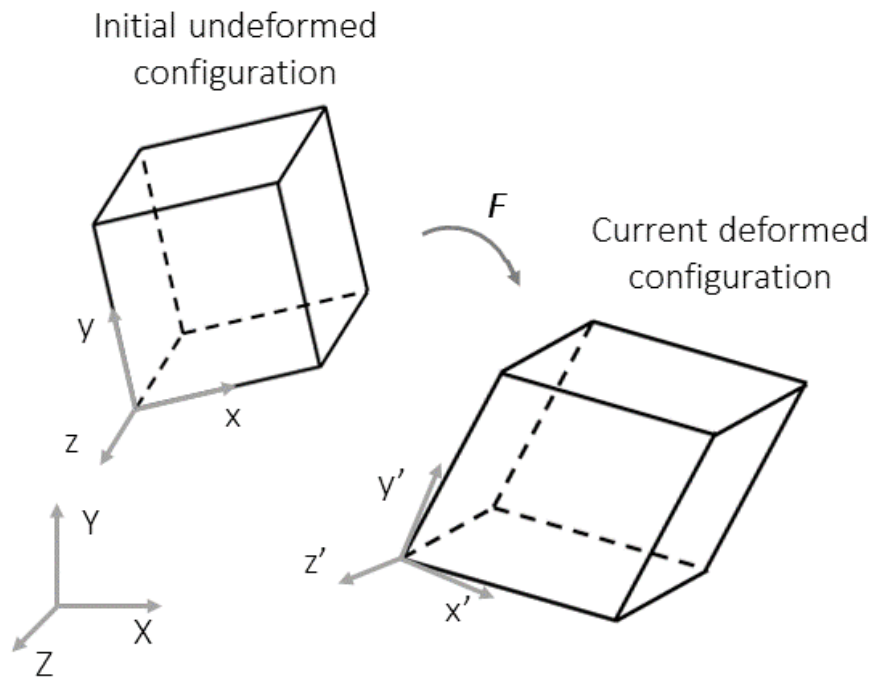

Figure 1: The three considered coordinate systems. $\mathrm{X}, \mathrm{Y}$ and $\mathrm{Z}$ denote the global coordinate system, $\mathrm{x}, \mathrm{y}$ and $\mathrm{z}$ the initial local coordinate system and $\mathrm{x}^{\prime}, \mathrm{y}^{\prime}$ and $\mathrm{z}^{\prime}$ the current local coordinate system. denoted by x'y'z'. This local coordinate system is attached to the element and rotates with the deformations and rigid body rotations.

Using local orientations is practical in some situations, for example in a tubular structure, where due to material properties (e.g. fibers in the wall) or 
boundary conditions the use of a local cylindrical coordinate system is more convenient than a global rectangular coordinate system.

The user can decide to use a local coordinate system for the calculations by using the keyword *orientation in the Abaqus input file, and assigning the defined local coordinate system to one or multiple sections of the model.

- Global: Without the *orientation keyword, Abaqus uses the global coordinate system XYZ.

- Local: With the *orientation keyword and correct section assignment, Abaqus uses the current local coordinate system x'y'z', based on the initial local coordinate system xyz that was defined with this keyword.

\subsection{Material description and other prerequisites}

The GOH model as presented by [1] and also described in the Abaqus Analysis User's Guide 22.5.3 [2] uses a nearly incompressible, hyperelastic formulation to describe the mechanical behavior of arterial tissue. The strain energy density function $\Psi$ uses additive decomposition to describe the volumetric $\left(\Psi^{v o l}\right)$ and deviatoric part $\left(\Psi^{d e v}\right)$. The deviatoric strain energy in turn contains a contribution of extracellular matrix material and embedded collagen fibers. The matrix material $\left(\Psi^{i s o}\right)$ consists mainly of elastin which exhibits isotropic and linear behavior. The collagen fibers $\left(\Psi^{a n i}\right)$ are assumed to run along two preferred directions and exhibit an anisotropic and nonlinear behavior.

$$
\begin{aligned}
\Psi & =\Psi^{\text {dev }}+\Psi^{v o l}=\Psi^{i s o}+\Psi^{a n i}+\Psi^{v o l}, \\
\Psi^{i s o} & =C_{10}\left(\bar{I}_{1}-3\right), \\
\Psi^{a n i} & =\frac{k_{1}}{2 k_{2}} \sum_{i=4,6}\left\{\exp \left\{k_{2}\left[\kappa \bar{I}_{1}+(1-3 \kappa) \bar{I}_{i}-1\right]^{2}\right\}-1\right\}, \\
& =\frac{k_{1}}{2 k_{2}} \sum_{i=4,6}\left\{\exp \left\{k_{2} \bar{E}_{i}^{2}\right\}-1\right\}, \\
\bar{E}_{i} & =\kappa \bar{I}_{1}+(1-3 \kappa) \bar{I}_{i}-1 \text { for } i=4,6 . \\
\Psi^{v o l} & =\frac{1}{D}\left(\frac{J^{2}-1}{2}-\ln J\right),
\end{aligned}
$$


with $C_{10}, k_{1}, k_{2}, \kappa$ and $D$ material constants. $C_{10}$ and $k_{1}$ have positive values and

correspond to the matrix and fiber stiffness, respectively. Note that $C_{10}=\mu / 2$, in which $\mu$ is the more commonly known shear modulus. $k_{2}$ has a dimensionless positive value and is related to the nonlinearity of the fibers. $\kappa$ represents the dispersion of the fibers and has values between 0 (i.e. no dispersion) and $1 / 3$ (i.e. fully dispersed or isotropic). $D$ is a material parameter related to 85 the compressibility, i.e. $D=2 / K$ with $K$ the bulk modulus $\bar{I}_{1}$ is the first invariant of the isochoric right Cauchy-Green stretch tensor $\overline{\boldsymbol{C}}=\overline{\boldsymbol{F}}^{T} \overline{\boldsymbol{F}}$, with $\overline{\boldsymbol{F}}$ the isochoric part of the deformation gradient $\boldsymbol{F}$ calculated as $\overline{\boldsymbol{F}}=J^{-1 / 3} \boldsymbol{F}$, where $J$ is the Jacobian or determinant of the deformation gradient $\boldsymbol{F} . \bar{I}_{i}$ is a pseudo-invariant of $\overline{\boldsymbol{C}}$, and represents the stretch along each preferred fiber so direction as

$$
\bar{I}_{i}=M_{i}\left(\bar{C} M_{i}\right), \quad i=4,6,
$$

with $\boldsymbol{M}_{i}$ the undeformed mean fiber vector of each of the fiber families, expressed using material constant $\alpha_{i}$, which represents a mean fiber angle for the respective fiber family. Note that, in the version used here, the fiber contribution is activated only when $\bar{E}_{i}>0$.

\section{2.3. Material implementations}

The constitutive model described above can be used in the FE software Abaqus Standard in multiple ways. First, the GOH model is available in Abaqus as a built-in or pre-programmed material model. On the other hand, user subroutines such as UANISOHYPER_INV and UMAT allow the user to define a material model. These subroutines are written in Fortran with a level of complexity proportional to the extent of freedom to which the material can be defined. Below follows a discussion of these three main possibilities.

\subsubsection{Built-in implementation}

The GOH model is implemented as a built-in anisotropic, hyperelastic material, 'Holzapfel', in Abaqus (Abaqus Analysis User's Guide 22.5.3 [2]). The user needs to assign values to the material parameters and define the number 
of local directions or fiber families (i.e. two for the classic GOH model). Up to three fiber families can be defined and their fiber vectors need to be defined manually in the input file as local directions of the local coordinate system using the *orientation keyword. By default, all calculations are performed in the local coordinate system defined by the user.

\subsubsection{UANISOHYPER_INV implementation}

For invariant-based material formulations, such as the GOH model, Abaqus offers a user subroutine which requires the definition of the derivatives of the strain energy density function with respect to the invariants of the isochoric deformation gradient tensor and fiber directions (Abaqus User Subroutines Reference Guide 1.1.20 [2]). The most important variables passed in for information and the ones that need to be defined are shown in table 1 Again, up to three local directions and hence fiber families can be defined using the *orientation keyword and all calculations are performed in the local coordinate system defined by the user.

For the specific GOH model considered here, the first, second and third derivatives of the strain energy density function with respect to the invariants 
Table 1: Overview of the most important input and output variables in a UANISOHYPER_INV subroutine (Abaqus User Subroutines Reference Guide 1.1.20 [2]).

\begin{tabular}{|c|c|}
\hline VARIABLE & DESCRIPTION \\
\hline \multicolumn{2}{|l|}{ INPUT } \\
\hline NFIBERS & $\begin{array}{l}\text { Number of fiber families defined for this material in } \\
\text { the input file. }\end{array}$ \\
\hline NINV & $\begin{array}{l}\text { Number of scalar invariants. This depends on the } \\
\text { number of fiber families that is defined. E.g. in the } \\
\text { case of two fiber families, there is a total of } 9 \text { invari- } \\
\text { ants. }\end{array}$ \\
\hline PROPS & $\begin{array}{l}\text { Array of material properties entered for this user- } \\
\text { defined hyperelastic material. }\end{array}$ \\
\hline AINV(NINV) & $\begin{array}{l}\text { Array of scalar invariants, at each material point at } \\
\text { the end of the increment. }\end{array}$ \\
\hline \multicolumn{2}{|l|}{ Output } \\
\hline $\mathrm{UA}(1)$ & The strain energy density function $\Psi$. \\
\hline UI1(NINV) & $\begin{array}{l}\text { Array of first derivatives of the strain energy density } \\
\text { function with respect to the scalar invariants, } \partial \Psi / \partial \bar{I}_{i} \text {. }\end{array}$ \\
\hline $\mathrm{UI} 2\left(\mathrm{NINV}^{*}(\mathrm{NINV}+1) / 2\right)$ & $\begin{array}{l}\text { Array of second derivatives of the strain energy den- } \\
\text { sity function with respect to the scalar invariants, } \\
\partial^{2} \Psi / \partial \bar{I}_{i} \partial \bar{I}_{j} \text {. }\end{array}$ \\
\hline
\end{tabular}


(in casu $\bar{I}_{1}, \bar{I}_{4}, \bar{I}_{6}$ and $J$ ) are

$$
\begin{aligned}
\frac{\partial \Psi}{\partial \bar{I}_{1}} & =C_{10}+\sum_{i} k_{1} \bar{E}_{i} \exp \left(k_{2} \bar{E}_{i}^{2}\right) \kappa, \\
\frac{\partial^{2} \Psi}{\left(\partial \bar{I}_{1}\right)^{2}} & =k_{1} \exp \left(k_{2} \bar{E}_{i}^{2}\right) \kappa^{2}\left(2 k_{2} \bar{E}_{i}^{2}+1\right), \\
\frac{\partial \Psi}{\partial \bar{I}_{i}} & =k_{1} \bar{E}_{i} \exp \left(k_{2} \bar{E}_{i}^{2}\right)(1-3 \kappa), \\
\frac{\partial^{2} \Psi}{\left(\partial \bar{I}_{i}\right)^{2}} & =k_{1} \exp \left(k_{2} \bar{E}_{i}^{2}\right)(1-3 \kappa)^{2}\left(2 k_{2} \bar{E}_{i}^{2}+1\right), \\
\frac{\partial^{2} \Psi}{\partial \bar{I}_{1} \partial \bar{I}_{i}} & =k_{1} \exp \left(k_{2} \bar{E}_{i}^{2}\right)(1-3 \kappa) \kappa\left(2 k_{2} \bar{E}_{i}^{2}+1\right), \\
\frac{\partial \Psi}{\partial J} & =\frac{1}{D}\left(J-\frac{1}{J}\right), \\
\frac{\partial^{2} \Psi}{(\partial J)^{2}} & =\frac{1}{D}\left(1+\frac{1}{J^{2}}\right) \\
\frac{\partial^{3} \Psi}{(\partial J)^{3}} & =\frac{1}{D} \frac{-2}{J^{3}}
\end{aligned}
$$

125

for $i=4,6$.

In case of an incompressible hybrid formulation, the derivatives with respect to $J$ are ignored. In this case, add TYPE=INCOMPRESSIBLE to *anisotropic hyperelastic in the input file (Abaqus Keywords Reference Guide [2]).

\subsubsection{UMAT implementation}

130

A material behavior can also be defined in Abaqus using a user material subroutine or UMAT (Abaqus User Subroutines Reference Guide 1.1.41 [2]). This approach allows more freedom in defining a material model (e.g. more than three fiber families can be defined), but accordingly, the required user input is more complex. The Cauchy stress tensor and tangent stiffness matrix need to be defined, which for a hyperelastic material such as the GOH material model can be defined as a function of the deformation gradient. An overview of the most important variables passed in for information and the ones that need to be defined are shown in table 2 .

Without the *orientation keyword, these quantities must all be defined 
Table 2: Overview of the most important input and output variables in a UMAT subroutine (Abaqus User Subroutines Reference Guide 1.1.20 [2]).

\begin{tabular}{|c|c|}
\hline VARIABLE & DESCRIPTION \\
\hline \multicolumn{2}{|l|}{ INPUT } \\
\hline PROPS & $\begin{array}{l}\text { User-specified array of material constants associated with this } \\
\text { user material. }\end{array}$ \\
\hline DFGRD0 $(3,3)$ & $\begin{array}{l}\text { Array containing the deformation gradient at the beginning of } \\
\text { the increment. }\end{array}$ \\
\hline DFGRD1(3,3) & $\begin{array}{l}\text { Array containing the deformation gradient at the end of the in- } \\
\text { crement. }\end{array}$ \\
\hline \multicolumn{2}{|l|}{ OutPut } \\
\hline STRESS(d) & $\begin{array}{l}\text { This array is passed in as the stress tensor at the beginning of } \\
\text { the increment and must be updated in this routine to become the } \\
\text { stress tensor at the end of the increment. The measure of stress } \\
\text { used is Cauchy stress. For } 2 \mathrm{D} \text { and } 3 \mathrm{D} \text { elements, d is } 3 \text { and } 6 \\
\text { respectively. }\end{array}$ \\
\hline $\operatorname{DDSDDE}(\mathrm{d}, \mathrm{d})$ & $\begin{array}{l}\text { Tangent stiffness matrix of the constitutive model, } \frac{\partial \Delta \sigma}{\partial \Delta \epsilon} \text {, where } \\
\Delta \sigma \text { are the stress increments and } \Delta \epsilon \text { are the strain increments. } \\
\text { DDSDDE }(\mathrm{I}, \mathrm{J}) \text { defines the change in the Ith stress component at } \\
\text { the end of the time increment caused by an infinitesimal pertur- } \\
\text { bation of the Jth component of the strain increment array. For } \\
2 \mathrm{D} \text { and } 3 \mathrm{D} \text { elements, } \mathrm{d} \text { is } 3 \text { and } 6 \text { respectively. }\end{array}$ \\
\hline
\end{tabular}



word on the other hand, requires that the stress and tangent stiffness tensors are expressed in the current local coordinate system x'y'z'. In that case, the deformation gradient should logically map vectors from the undeformed configuration expressed in the initial local coordinate system to the deformed configuration expressed in the current local coordinate system (denoted as $\boldsymbol{F}^{x y z \rightarrow x^{\prime} y^{\prime} z^{\prime}}$ ). This is often convenient since fiber vectors are usually known in the initial configuration. For shell and membrane elements, this is exactly what is done in Abaqus. However, for solid continuum elements, Abaqus (for all versions of Abaqus at least up to version 2019) passes in a deformation gradient that maps vectors from the undeformed configuration expressed in the current local coordinate system to the deformed configuration expressed in the same current local coordinate system (denoted as $\boldsymbol{F}^{x^{\prime} y^{\prime} z^{\prime} \rightarrow x^{\prime} y^{\prime} z^{\prime}}$ ). Working with this deformation gradient for our further calculations implies that also the fiber vectors need to be defined in the current local coordinate system or that the deformation gradient of local orientations for solid continuum elements requires an intermediate step. More details about this can be found in brief in the Appendix 8.1, and more elaborately in [3]. In the remainder of this section, the deformation gradient is simply denoted as $\boldsymbol{F}$, where it can refer to either $\boldsymbol{F}^{x y z \rightarrow x^{\prime} y^{\prime} z^{\prime}}$ or $\boldsymbol{F}^{X Y Z \rightarrow X Y Z}$, the deformation gradient in the global coordinate system.

\subsubsection{Cauchy stress calculation}

\section{General}

For hyperelastic materials, the second Piola-Kirchhoff stress $\boldsymbol{S}$ is twice the derivative of the strain energy density function with respect to the right Cauchy-

165 Green stretch tensor $\boldsymbol{C}$, i.e.

$$
\boldsymbol{S}=2 \frac{\partial \Psi}{\partial \boldsymbol{C}}
$$

Applying the push-forward operation to obtain the Cauchy stress $\boldsymbol{\sigma}$ yields 
[4, 5]:

$$
\boldsymbol{\sigma}=\frac{1}{J} \boldsymbol{F} \boldsymbol{S} \boldsymbol{F}^{T}=\frac{2}{J} \boldsymbol{F} \frac{\partial \Psi}{\partial \boldsymbol{C}} \boldsymbol{F}^{T} .
$$

Using $\boldsymbol{C}=\boldsymbol{F}^{T} \boldsymbol{F}$ and (see appendix 8.3)

$$
\frac{\partial \Psi}{\partial \boldsymbol{F}}=2 \boldsymbol{F} \frac{\partial \Psi}{\partial \boldsymbol{C}},
$$

this can be rewritten as

$$
\boldsymbol{\sigma}=\frac{1}{J} \frac{\partial \Psi}{\partial \boldsymbol{F}} \boldsymbol{F}^{T}
$$

or in index notation,

$$
\sigma_{i j}=\frac{1}{J} \frac{\partial \Psi}{\partial F_{i a}} F_{j a} .
$$

In order to derive the Cauchy stress for the GOH model according to equation 8 , bear in mind that

$$
\begin{aligned}
\frac{\partial I_{1}}{\partial F_{i a}} & =2 F_{i a}, \\
\frac{\partial I_{4,6}}{\partial F_{i a}} & =2(\boldsymbol{F} \boldsymbol{M})_{i} M_{a}, \\
\frac{\partial J}{\partial F_{i a}} & =J\left(\boldsymbol{F}^{-T}\right)_{i a}, \\
\frac{\partial \bar{I}_{1}}{\partial F_{i a}} & =J^{-2 / 3}\left(2 F_{i a}-\frac{2}{3}\left(\boldsymbol{F}^{-T}\right)_{i a} I_{1}\right), \text { and } \\
\frac{\partial \bar{I}_{4,6}}{\partial F_{i a}} & =J^{-2 / 3}\left(2(\boldsymbol{F} \boldsymbol{M})_{i} M_{a}-\frac{2}{3}\left(\boldsymbol{F}^{-T}\right)_{i a} I_{4,6}\right) .
\end{aligned}
$$

\section{Solid continuum elements}

For the GOH model, the Cauchy stress tensor then becomes

$$
\begin{aligned}
\sigma_{i j}^{i s o} & =\frac{1}{J} \frac{\partial \Psi^{i s o}}{\partial \bar{I}_{1}} \frac{\partial \bar{I}_{1}}{\partial F_{i a}} F_{j a} \\
& =\frac{2}{J} C_{10}\left(\overline{\boldsymbol{B}}_{i j}-\frac{1}{3} \delta_{i j} \bar{I}_{1}\right), \\
\sigma_{i j}^{a n i} & =\frac{1}{J}\left(\frac{\partial \Psi^{a n i}}{\partial \bar{I}_{1}} \frac{\partial \bar{I}_{1}}{\partial F_{i a}}+\frac{\partial \Psi^{a n i}}{\partial \bar{I}_{4}} \frac{\partial \bar{I}_{4}}{\partial F_{i a}}\right) F_{j a} \\
& =\sum \frac{2 k_{1}}{J} \exp \left(k_{2} \bar{E}^{2}\right) \bar{E}\left[\kappa\left(\bar{B}_{i j}-\frac{1}{3} \delta_{i j} \bar{I}_{1}\right)+(1-3 \kappa)\left(\bar{m}_{i} \bar{m}_{j}-\frac{1}{3} \delta_{i j} \bar{I}_{4}\right)\right], \\
\sigma_{i j}^{v o l} & =\frac{1}{J} \frac{\partial \Psi^{v o l}}{\partial J} \frac{\partial J}{\partial F_{i a}} F_{j a} \\
& =\frac{1}{D}\left(J-\frac{1}{J}\right) \delta_{i j},
\end{aligned}
$$




\section{Plane stress shell and membrane elements}

Shell and membrane elements are two ways to model a plane stress situation, for which the stress component normal to the $2 \mathrm{D}$ surface of the element must be equal to zero. In the current description, we will focus on the fully incompressible case, which is also the only option that Abaqus provides for these elements. In that case, the deformation gradient passed through the variable DFGRD1 to the UMAT subroutine is of the form

$$
\boldsymbol{F}=\left[\begin{array}{lll}
F_{11} & F_{12} & F_{13} \\
F_{21} & F_{22} & F_{23} \\
F_{31} & F_{32} & F_{33}
\end{array}\right]=\left[\begin{array}{ccc}
F_{11} & F_{12} & 0 \\
F_{21} & F_{22} & 0 \\
0 & 0 & \frac{1}{F_{11} F_{22}-F_{12} F_{21}}
\end{array}\right]
$$

To obtain a zero normal Cauchy stress $\left(\sigma_{33}=0\right)$, a hydrostatic pressure $p$ must be added to the Cauchy stress [4, 5]

$$
\sigma_{i j}=\sigma_{i j}^{i s o}+\sigma_{i j}^{a n i}-p \delta_{i j}
$$

By decomposing $p$ in $p^{i s o}$ and $p^{a n i}$ and stating that $p^{i s o}=\sigma_{33}^{i s o}$ and $p^{a n i}=\sigma_{33}^{a n i}$, the following expressions for stress remain:

$$
\begin{aligned}
\sigma_{i j}^{i s o}-p^{i s o} \delta_{i j} & =2 C_{10}\left(B_{i j}-B_{33} \delta_{i j}\right) \\
\sigma_{i j}^{a n i}-p^{a n i} \delta_{i j} & =\sum 2 k_{1} \exp \left(k_{2} E^{2}\right) E\left[\kappa\left(B_{i j}-B_{33} \delta_{i j}\right)+(1-3 \kappa)\left(m_{i} m_{j}-m_{3} m_{3} \delta_{i j}\right)\right]
\end{aligned}
$$

Note that in this incompressible case $J=1$, such that the deformation is fully isochoric. 
The variable STRESS to be defined in the UMAT contains the three independent Cauchy stress components in the following order: $\sigma_{11}, \sigma_{22}$ and $\sigma_{12}$.

\subsubsection{Tangent stiffness matrix calculation} literature alternative expressions have been proposed, such as [6]

$$
\mathbb{C}_{i j k l}=\sigma_{i j} \delta_{k l}+\frac{1}{2}\left(\frac{\partial \sigma_{i j}}{\partial F_{k a}} F_{l a}+\frac{\partial \sigma_{i j}}{\partial F_{l a}} F_{k a}\right),
$$

and

$$
\mathbb{C}_{i j k l}=\frac{2}{J} \frac{\partial S_{a b}}{\partial C_{c d}} F_{i a} F_{j b} F_{k c} F_{l d}+\frac{1}{2}\left(\delta_{i k} \sigma_{j l}+\delta_{i l} \sigma_{j k}+\delta_{j k} \sigma_{i l}+\delta_{j l} \sigma_{i k}\right),
$$

for example in [7, 8].

The four expressions for the tangent stiffness matrix, given in equations 14 215 15, 16 and 17 are equivalent up to small assumptions. This is further elaborated in appendix 8.2 .

\section{Solid continuum elements}


We can now apply the formulation given in equation 16 to the constitutive model defined in equation 1 . The Cauchy stress expressions are given in equation 10. Knowing that

$$
\begin{aligned}
\frac{\partial B_{i j}}{\partial F_{k a}} & =\delta_{i k} F_{j a}+\delta_{j k} F_{i a} \\
\frac{\partial \bar{B}_{i j}}{\partial F_{k a}} & =J^{-2 / 3}\left(\delta_{i k} F_{j a}+\delta_{j k} F_{i a}\right)-\frac{2}{3} J^{-2 / 3}\left(\boldsymbol{F}^{-T}\right)_{k a} B_{i j} \\
\frac{\partial \bar{B}_{i j}}{\partial F_{k a}} F_{l a} & =\delta_{i k} \bar{B}_{j l}+\delta_{j k} \bar{B}_{i l}-\frac{2}{3} \delta_{k l} \bar{B}_{i j} \\
\frac{\partial\left(m_{i} m_{j}\right)}{\partial F_{k a}} & =\delta_{i k} M_{a} m_{j}+\delta_{j k} M_{a} m_{i} \\
\frac{\partial\left(\bar{m}_{i} \bar{m}_{j}\right)}{\partial F_{k a}} & =J^{-2 / 3}\left(\delta_{i k} M_{a} m_{j}+\delta_{j k} M_{a} m_{i}\right)-\frac{2}{3} J^{-2 / 3}\left(\boldsymbol{F}^{-T}\right)_{k a} m_{i} m_{j} \\
\frac{\partial\left(\bar{m}_{i} \bar{m}_{j}\right)}{\partial F_{k a}} F_{l a} & =\delta_{i k} \bar{m}_{l} \bar{m}_{j}+\delta_{j k} \bar{m}_{l} \bar{m}_{i}-\frac{2}{3} \delta_{k l} \bar{m}_{i} \bar{m}_{j},
\end{aligned}
$$

and using equations 16 or 17 the expressions for the tangent stiffness matrices are

$$
\begin{aligned}
\mathbb{C}_{i j k l}^{i s o} & =\frac{2}{J} C_{10}\left(\frac{-2}{3} \delta_{k l} \bar{B}_{i j}+\frac{2}{9} \delta_{i j} \delta_{k l} \bar{I}_{1}+\frac{1}{2}\left(\delta_{i l} \bar{B}_{k j}+\delta_{j l} \bar{B}_{i k}+\delta_{i k} \bar{B}_{l j}+\delta_{j k} \bar{B}_{i l}\right)\right. \\
& \left.-\frac{1}{3}\left(\delta_{i j} \bar{B}_{l k}+\delta_{i j} \bar{B}_{k l}\right)\right) \\
\mathbb{C}_{i j k l}^{a n i} & =\sum \frac{2 k_{1}}{J} \exp \left(k_{2} \bar{E}^{2}\right)\left\{\bar{E} \kappa\left(\bar{B}_{i j} \delta_{k l}-\frac{1}{3} \delta_{i j} \delta_{k l} \bar{I}_{1 C}\right)+\bar{E}(1-3 \kappa)\left(\bar{m}_{i} \bar{m}_{j} \delta_{k l}-\frac{1}{3} \delta_{i j} \delta_{k l} \bar{I}_{4}\right)\right. \\
& +\left[\frac{-5}{3} \bar{E} \kappa \delta_{k l}+\left(2 k_{2} \bar{E}^{2}+1\right) \kappa^{2}\left(\bar{B}_{k l}+\bar{B}_{l k}-\frac{2}{3} \delta_{k l} \bar{I}_{1}\right)\right]\left(\bar{B}_{i j}-\frac{1}{3} \bar{I}_{1} \delta_{i j}\right) \\
& +\bar{E} \kappa\left[\frac{1}{2}\left(\delta_{i k} \bar{B}_{l j}+\delta_{j k} \bar{B}_{i l}+\delta_{i l} \bar{B}_{k j}+\delta_{j l} \bar{B}_{i k}\right)-\frac{1}{3}\left(\bar{B}_{k l} \delta_{i j}+\bar{B}_{l k} \delta_{i j}\right)\right] \\
& +\left[\frac{-5}{3} \bar{E}(1-3 \kappa) \delta_{k l}+\left(2 k_{2} \bar{E}^{2}+1\right)(1-3 \kappa)^{2}\left(2 \bar{m}_{k} \bar{m}_{l}-\frac{2}{3} \delta_{k l} \bar{I}_{4 C}\right)\right]\left(\bar{m}_{i} \bar{m}_{j}-\frac{1}{3} \bar{I}_{4} \delta_{i j}\right) \\
& \left.+\bar{E}(1-3 \kappa)\left[\frac{1}{2}\left(\delta_{i k} \bar{m}_{l} \bar{m}_{j}+\delta_{j k} \bar{m}_{i} \bar{m}_{l}+\delta_{i l} \bar{m}_{k} \bar{m}_{j}+\delta_{j l} \bar{m}_{i} \bar{m}_{k}\right)-\frac{2}{3} \delta_{i j} \bar{m}_{k} \bar{m}_{l}\right]\right\} \\
\mathbb{C}_{i j k l}^{v o l} & =\frac{2}{D J} \delta_{i j} \delta_{k l} .
\end{aligned}
$$

This fourth order tensor contains major and minor symmetry, such that 36 independent components must be returned through the variable DDSDDE in a 6 
by 6 matrix as

$$
\left[\begin{array}{llllll}
\mathbb{C}_{1111} & \mathbb{C}_{1122} & \mathbb{C}_{1133} & \mathbb{C}_{1112} & \mathbb{C}_{1113} & \mathbb{C}_{1123} \\
\mathbb{C}_{2211} & \mathbb{C}_{2222} & \mathbb{C}_{2233} & \mathbb{C}_{2212} & \mathbb{C}_{2213} & \mathbb{C}_{2223} \\
\mathbb{C}_{3311} & \mathbb{C}_{3322} & \mathbb{C}_{3333} & \mathbb{C}_{3312} & \mathbb{C}_{3313} & \mathbb{C}_{3323} \\
\mathbb{C}_{1211} & \mathbb{C}_{1222} & \mathbb{C}_{1233} & \mathbb{C}_{1212} & \mathbb{C}_{1213} & \mathbb{C}_{1223} \\
\mathbb{C}_{1311} & \mathbb{C}_{1322} & \mathbb{C}_{1333} & \mathbb{C}_{1312} & \mathbb{C}_{1313} & \mathbb{C}_{1323} \\
\mathbb{C}_{2311} & \mathbb{C}_{2322} & \mathbb{C}_{2333} & \mathbb{C}_{2312} & \mathbb{C}_{2313} & \mathbb{C}_{2323}
\end{array}\right]
$$

\section{Plane stress shell and membrane elements}

Recall from equation 11 that we only have four independent components in the deformation gradient for plane stress elements. Therefore $\frac{\partial F_{i j}}{\partial F_{k l}}=\delta_{i k} \delta_{j l}$ is no longer generally true. Instead we write

$$
\begin{aligned}
& \frac{\partial F_{33}}{\partial F_{11}}=-F_{22} F_{33}^{2} \\
& \frac{\partial F_{33}}{\partial F_{22}}=-F_{11} F_{33}^{2} \\
& \frac{\partial F_{33}}{\partial F_{12}}=F_{21} F_{33}^{2} \\
& \frac{\partial F_{33}}{\partial F_{21}}=F_{12} F_{33}^{2}
\end{aligned}
$$

The derivatives of the invariants can be written as

$$
\begin{aligned}
\frac{\partial I_{1}}{\partial F_{11}} & =2 F_{11}-2 F_{22} F_{33}^{3} \\
\frac{\partial I_{1}}{\partial F_{22}} & =2 F_{22}-2 F_{11} F_{33}^{3} \\
\frac{\partial I_{1}}{\partial F_{12}} & =2 F_{12}+2 F_{21} F_{33}^{3} \\
\frac{\partial I_{1}}{\partial F_{21}} & =2 F_{21}+2 F_{12} F_{33}^{3} \\
\frac{\partial I_{1}}{\partial F_{33}} & =2 F_{33}
\end{aligned}
$$

and as

$$
\frac{\partial I_{i}}{\partial F_{k a}}=M_{b} \frac{\partial F_{c b}}{\partial F_{k a}} m_{c}+m_{c} \frac{\partial F_{c d}}{\partial F_{k a}} M_{d} \text { for } i=4,6
$$


Applying equation 16 to the Cauchy stress from equation 13 , gives

$$
\begin{aligned}
\mathbb{C}_{i j k l}^{i s o} & =2 C_{10}\left[B_{i j} \delta_{k l}-B_{33} \delta_{i j} \delta_{k l}+\frac{1}{2}\left(\frac{\partial F_{i b}}{\partial F_{k a}} F_{j b} F_{l a}+F_{i b} \frac{\partial F_{j b}}{\partial F_{k a}} F_{l a}\right.\right. \\
& \left.\left.+\frac{\partial F_{i b}}{\partial F_{l a}} F_{j b} F_{k a}+F_{i b} \frac{\partial F_{j b}}{\partial F_{l a}} F_{k a}\right)-F_{33} \frac{\partial F_{33}}{\partial F_{k a}} F_{l a} \delta_{i j}-F_{33} \frac{\partial F_{33}}{\partial F_{l a}} F_{k a} \delta_{i j}\right] \\
\mathbb{C}_{i j k l}^{a n i} & =2 k_{1} \exp \left(k_{2} E^{2}\right) E\left[\kappa\left(B_{i j}-B_{33} \delta_{i j}\right)+(1-3 \kappa)\left(m_{i} m_{j}-m_{3} m_{3} \delta_{i j}\right)\right] \\
& {\left[E \delta_{k l}+\frac{1}{2}\left(2 k_{2} E^{2}+1\right)\left(\frac{\partial E}{\partial F_{k a}} F_{l a}+\frac{\partial E}{\partial F_{l a}} F_{k a}\right)\right] } \\
& +k_{1} \exp \left(k_{2} E^{2}\right) E\left[\kappa \left(\frac{\partial F_{i b}}{\partial F_{k a}} F_{j b} F_{l a}+\frac{\partial F_{i b}}{\partial F_{l a}} F_{j b} F_{k a}+\frac{\partial F_{j b}}{\partial F_{k a}} F_{i b} F_{l a}+\frac{\partial F_{j b}}{\partial F_{l a}} F_{i b} F_{k a}\right.\right. \\
& \left.-2 F_{33} \frac{\partial F_{33}}{\partial F_{k a}} \delta_{i j} F_{l a}-2 F_{33} \frac{\partial F_{33}}{\partial F_{l a}} \delta_{i j} F_{k a}\right)+(1-3 \kappa)\left(\frac{\partial F_{i b}}{\partial F_{k a}} M_{b} m_{j} F_{l a}+\frac{\partial F_{i b}}{\partial F_{l a}} M_{b} m_{j} F_{k a}\right. \\
& \left.\left.+\frac{\partial F_{j b}}{\partial F_{k a}} M_{b} m_{i} F_{l a}+\frac{\partial F_{j b}}{\partial F_{l a}} M_{b} m_{i} F_{k a}-2 \frac{\partial F_{3 b}}{\partial F_{k a}} M_{b} m_{3} F_{l a} \delta_{i j}-2 \frac{\partial F_{3 b}}{\partial F_{l a}} M_{b} m_{3} F_{k a} \delta_{i j}\right)\right],
\end{aligned}
$$

where $\frac{\partial E}{\partial F_{k a}}=\kappa \frac{\partial I_{1}}{\partial F_{k a}}+(1-3 \kappa) \frac{\partial I_{4}}{\partial F_{k a}}$.

For plane stress elements, DDSDDE should be returned as

$$
\left[\begin{array}{lll}
\mathbb{C}_{1111} & \mathbb{C}_{1122} & \mathbb{C}_{1112} \\
\mathbb{C}_{2211} & \mathbb{C}_{2222} & \mathbb{C}_{2212} \\
\mathbb{C}_{1211} & \mathbb{C}_{1222} & \mathbb{C}_{1212}
\end{array}\right]
$$

\section{Numerical derivation}

The tangent stiffness tensor can be calculated analytically, as outlined above, or approximated numerically [7, 8, 9]. The method is based on the application of small perturbations in all directions of the deformation gradient and retrieving the resulting change in stress. A more in-depth explanation can be found in [9].

The perturbed deformation gradient in only its $(i, j)$ component may be written as

$$
\hat{\boldsymbol{F}}^{(i j)}=\boldsymbol{F}+\boldsymbol{\Delta} \boldsymbol{F}^{(i j)},
$$

where

$$
\Delta \boldsymbol{F}^{(i j)}=\frac{\epsilon}{2}\left(\boldsymbol{e}_{i} \otimes \boldsymbol{e}_{j} \boldsymbol{F}+\boldsymbol{e}_{j} \otimes \boldsymbol{e}_{i} \boldsymbol{F}\right)
$$


and $\epsilon$ is a small perturbation parameter equal to $2 \cdot 10^{-8}$ in the current analyses. The numerically derived tangent stiffness is then

$$
\mathbb{C}_{i j k l}=\frac{1}{J \epsilon}\left[J_{p}^{(i j)} \boldsymbol{\sigma}\left(\hat{\boldsymbol{F}}^{(i j)}\right)-J \boldsymbol{\sigma}(\boldsymbol{F})\right]_{k l},
$$

245 where $J=\operatorname{det}(\boldsymbol{F}), J_{p}^{(i j)}=\operatorname{det}\left(\hat{\boldsymbol{F}}^{(i j)}\right)$ and $\boldsymbol{\sigma}$ is the total Cauchy stress.

This numerical approach can be used in combination with any element type. However, remember that for shell and membrane elements $F_{33}$ depends on the four other components of the deformation gradient, as given in equation 11 . Therefore, any perturbation in one of these components must cause a perturbation in $F_{33}$ as well. This can be achieved by setting

$$
\left(\hat{\boldsymbol{F}}^{(i j)}\right)_{33}=\frac{1}{\left(\hat{\boldsymbol{F}}^{(i j)}\right)_{11}\left(\hat{\boldsymbol{F}}^{(i j)}\right)_{22}-\left(\hat{\boldsymbol{F}}^{(i j)}\right)_{12}\left(\hat{\boldsymbol{F}}^{(i j)}\right)_{21}} .
$$

\subsection{Test cases}

All of the above implementations of the GOH material were tested and compared on a single element for solid continuum and plane stress elements. Four test cases can be identified, in which the *orientation keyword is used:

- Case BIL: Built-In hyperelastic anisotropic Holzapfel implementation in a Local coordinate system

- Case UIL: Uanisohyper_Inv implementation in a Local coordinate system

- Case UAL: Umat implementation in a Local coordinate system with Analytical tangent stiffness

- Case UNL: Umat implementation in a Local coordinate system with Numerical tangent stiffness

Two additional test cases are identified when using a UMAT with solid elements and the *orientation keyword is omitted (note that it is not possible to omit this keyword when using plane stress elements and solid elements in the BI and UI cases): 
- Case UAG: Umat implementation in a Global coordinate system with Analytical tangent stiffness

- Case UNG: Umat implementation in a Global coordinate system with Numerical tangent stiffness

Each of these test cases was subjected to the same set of deformations, including tension, shear and compression.

The used material parameters correspond to sheep aortic tissue [10] and are 275 shown in Table 3 .

\begin{tabular}{|l|c|}
\hline$C_{10}[\mathrm{MPa}]$ & 0.0305 \\
$D[\mathrm{MPa}]$ & 0.0 or 10.17 \\
$k_{1}[\mathrm{MPa}]$ & 0.0289 \\
$k_{2}[-]$ & 1.2315 \\
$\kappa[-]$ & 0.2764 \\
$\alpha[\mathrm{RAD}]$ & 1.5690 \\
\hline
\end{tabular}

Table 3: GOH material parameters used in all simulations.

Note that for solid elements both an incompressible and nearly incompressible material was considered. For the compressible material, the inverse of the bulk modulus $D$ was calculated using the following formula:

$$
D=\frac{2 C_{10}}{3(1-2 \nu)},
$$

with a Poisson ratio $\nu=0.499$. For plane stress elements, only the incompressible case was considered.

The two collagen fiber families were assumed to be symmetric around the $\mathrm{X}$-direction and could therefore be characterized with $\alpha$ and $-\alpha$. When a local coordinate system was used, its initial orientation was the same as the global coordinate system. Hence, the undeformed fiber vector $\boldsymbol{M}_{i}$ was defined as

$$
\boldsymbol{M}_{i}=\left[\begin{array}{ll}
\cos \alpha & \sin \alpha
\end{array}\right],
$$


where $\alpha$ was defined with respect to the $\mathrm{X}$-axis.

\subsubsection{Single solid continuum element}

For solid continuum elements a full integration, hybrid, hexahedral element C3D8H was used (solid incompressible case) or a nonhybrid element C3D8 (solid

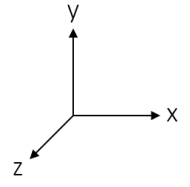

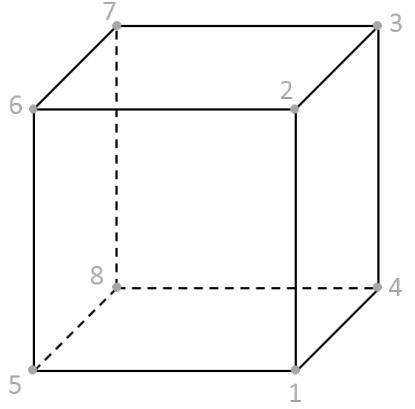

\begin{tabular}{l|l} 
Surface & Nodes \\
\hline S1 & $1,2,3,4$ \\
S2 & $5,6,7,8$ \\
S3 & $1,2,5,6$ \\
S4 & $2,3,6,7$ \\
S5 & $3,4,7,8$ \\
S6 & $1,4,5,8$
\end{tabular}

Figure 2: The 1-element cube with the relevant surfaces and corresponding nodes.

The sequence of simulation steps and corresponding boundary conditions are shown in table 4 where $t=10.0 \mathrm{~mm}, s=10.0 \mathrm{~mm}$ and $c=0.04 \mathrm{MPa}$.

\subsubsection{Single plane stress membrane element}

For plane stress elements the four implementations were tested on a single full integration quadrilateral membrane element M3D4. The element had edges of $50 \mathrm{~mm}$ and a thickness of $10 \mathrm{~mm}$. The thin square element, its relevant surfaces and corresponding nodes are shown in Figure 3.

The sequence of simulation steps and corresponding boundary conditions are shown in table 5 , where $s=10.0 \mathrm{~mm}, t=10.0 \mathrm{~mm}$ and $c=10.0 \mathrm{MPa}$.

\subsection{Comparison of results of single element test cases}

From each simulation, the resulting Cauchy stresses, displacements and reaction forces in every node were extracted for every step. All results were returned in the global coordinate system. In addition, the required number of iterations 


\begin{tabular}{|c|c|c|c|}
\hline State & Sketch & Boundary conditions & Step \\
\hline Stretch Y & & $\begin{array}{l}\text { Fix } \mathrm{S} 1 \text { in } \mathrm{X}, \text { Fix } \mathrm{S} 6 \text { in } \mathrm{Y}, \text { Fix } \mathrm{S} 3 \\
\text { in } \mathrm{Z}, \text { Move } \mathrm{S} 4 \text { in } \mathrm{Y} t\end{array}$ & $\begin{array}{l}\text { Step 1: Stretch Y } \\
\text { Step 2: Release Stretch Y }\end{array}$ \\
\hline Stretch Z & & $\begin{array}{l}\text { Fix } \mathrm{S} 1 \text { in } \mathrm{X}, \text { Fix } \mathrm{S} 6 \text { in } \mathrm{Y}, \text { Fix } \mathrm{S} 3 \\
\text { in } \mathrm{Z}, \text { Move } \mathrm{S} 5 \text { in } \mathrm{Z} t\end{array}$ & $\begin{array}{l}\text { Step 3: Stretch Z } \\
\text { Step 4: Release Stretch Z }\end{array}$ \\
\hline Stretch X & & $\begin{array}{l}\text { Fix } \mathrm{S} 1 \text { in } \mathrm{X}, \text { Fix } \mathrm{S} 6 \text { in } \mathrm{Y}, \text { Fix } \mathrm{S} 3 \\
\text { in } \mathrm{Z}, \text { Move } \mathrm{S} 2 \text { in } \mathrm{X} t\end{array}$ & $\begin{array}{l}\text { Step 5: Stretch X } \\
\text { Step 6: Release Stretch X }\end{array}$ \\
\hline Shear XY & & $\begin{array}{l}\text { Fix } \mathrm{S} 4 \text { in } \mathrm{Y}, \text { Fix } \mathrm{S} 5 \text { in } \mathrm{Z}, \mathrm{Fix} \mathrm{S} 6 \\
\text { in } \mathrm{X} \text { and } \mathrm{Y}, \text { Move } \mathrm{S} 4 \text { in } \mathrm{X} s\end{array}$ & $\begin{array}{l}\text { Step 7: Shear YX } \\
\text { Step 8: Release Shear YX }\end{array}$ \\
\hline Shear XY & & $\begin{array}{l}\text { Fix } \mathrm{S} 1 \text { in } \mathrm{X} \text { and } \mathrm{Y}, \mathrm{Fix} \mathrm{S} 2 \text { in } \mathrm{X} \\
\text { Fix } \mathrm{S} 5 \text { in } \mathrm{Z}, \text { Move } \mathrm{S} 2 \text { in } \mathrm{Y} s\end{array}$ & $\begin{array}{l}\text { Step 9: Shear XY } \\
\text { Step 10: Release Shear XY }\end{array}$ \\
\hline Shear YZ & & $\begin{array}{l}\text { Fix } \mathrm{S} 2 \text { in } \mathrm{X}, \text { Fix } \mathrm{S} 4 \text { in } \mathrm{Y}, \text { Fix } \mathrm{S} 6 \\
\text { in } \mathrm{Y} \text { and } \mathrm{Z}, \text { Move } \mathrm{S} 4 \text { in } \mathrm{Z} s\end{array}$ & $\begin{array}{l}\text { Step 11: Shear YZ } \\
\text { Step 12: Release Shear YZ }\end{array}$ \\
\hline Shear ZY & & $\begin{array}{l}\text { Fix } \mathrm{S} 2 \text { in } \mathrm{X}, \text { Fix } \mathrm{S} 3 \text { in } \mathrm{Y} \text { and } \mathrm{Z}, \\
\text { Fix S5 in Z, Move } \mathrm{S} 5 \text { in } \mathrm{Y} s\end{array}$ & $\begin{array}{l}\text { Step 13: Shear ZY } \\
\text { Step 14: Release Shear ZY }\end{array}$ \\
\hline Shear XZ & & $\begin{array}{l}\text { Fix } \mathrm{S} 1 \text { in } \mathrm{X} \text { and } \mathrm{Z}, \mathrm{Fix} \mathrm{S} 2 \text { in } \mathrm{X}, \\
\text { Fix S6 in } \mathrm{Y}, \text { Move } \mathrm{S} 2 \text { in } \mathrm{Z} s\end{array}$ & $\begin{array}{l}\text { Step 15: Shear XZ } \\
\text { Step 16: Release Shear XZ }\end{array}$ \\
\hline Shear ZX & & $\begin{array}{l}\text { Fix } \mathrm{S} 3 \text { in } \mathrm{X} \text { and } \mathrm{Z}, \mathrm{Fix} \mathrm{S} 5 \text { in } \mathrm{Z}, \\
\text { Fix S6 in } \mathrm{Y} \text {, Move S5 in } \mathrm{X} s\end{array}$ & $\begin{array}{l}\text { Step 17: Shear ZX } \\
\text { Step 18: Release Shear ZX }\end{array}$ \\
\hline Compression Y & & $\begin{array}{l}\text { Fix } \mathrm{S} 1 \text { in } \mathrm{X}, \text { Fix } \mathrm{S} 3 \text { in } \mathrm{Z}, \mathrm{Fix} \mathrm{S} 6 \\
\text { in } \mathrm{Y}, \text { Load } \mathrm{S} 4 \text { in } \mathrm{Y} c\end{array}$ & $\begin{array}{l}\text { Step 19: Compression Y } \\
\text { Step 20: Release Compression Y }\end{array}$ \\
\hline Compression Z & & $\begin{array}{l}\text { Fix } \mathrm{S} 1 \text { in } \mathrm{X}, \text { Fix } \mathrm{S} 3 \text { in } \mathrm{Z}, \text { Fix } \mathrm{S} 6 \\
\text { in } \mathrm{Y}, \text { Load } \mathrm{S} 5 \text { in } \mathrm{Z} c\end{array}$ & $\begin{array}{l}\text { Step 21: Compression Z } \\
\text { Step 22: Release Compression Z }\end{array}$ \\
\hline Compression X & & $\begin{array}{l}\text { Fix } \mathrm{S} 1 \text { in } \mathrm{X}, \mathrm{Fix} \mathrm{S} 3 \text { in } \mathrm{Z}, \mathrm{Fix} \mathrm{S} 6 \\
\text { in } \mathrm{Y}, \text { Load } \mathrm{S} 2 \text { in } \mathrm{X} c\end{array}$ & $\begin{array}{l}\text { Step 23: Compression X } \\
\text { Step 24: Release Compression X }\end{array}$ \\
\hline
\end{tabular}

зо5 for the finite element solver, and the CPU time were tracked.

To compare the accuracy of the different implementations, the built-in im- 


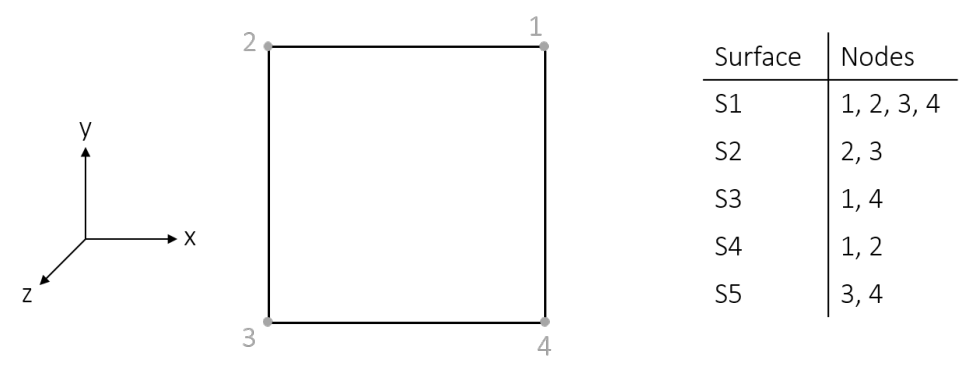

Figure 3: The 1-element thin square with the relevant surfaces and corresponding nodes.

plementation (Case BIL) was considered the reference case and the error of each component of the other test cases was calculated with respect to this reference. a length of $50 \mathrm{~mm}$, an inner radius of $10 \mathrm{~mm}$, and a wall thickness of 1.5 $\mathrm{mm}$. Due to symmetries in the central axial plane and a circumferential though the middle of the opening angle, only a fourth of the cylinder was accounted for in the model and symmetry boundary conditions were applied throughout 325

$$
T R E_{c, j}=\frac{\sqrt{\sum_{\text {nodes }} \sum_{\text {steps }}\left(c_{j}-c_{r}\right)^{2}}}{\sqrt{\sum_{\text {nodes steps }} c_{r}^{2}}},
$$

\subsubsection{Tests on multiple elements geometry}

To test the different material implementations on a geometry consisting of a more realistic number of elements with less homogeneous deformations, an extension-inflation test of an arterial segment was simulated. The stress-free configuration of the segment was considered a cylindrical section of $150^{\circ}$ with the simulation. In a first step, the opening angle was closed. The radial and 
Table 5: Simulation steps to test material implementations for the membrane element case.

\begin{tabular}{l|l|l|l} 
State & Sketch & Boundary conditions & Step \\
\hline Stretch Y & & $\begin{array}{l}\text { Fix S1 in Z, Fix S2 in X, Fix S5 } \\
\text { in Y, Move S4 in Y } t\end{array}$ & $\begin{array}{l}\text { Step 1: Stretch Y } \\
\text { Step 2: Release Stretch Y }\end{array}$ \\
\hline Stretch X & & $\begin{array}{l}\text { Fix S1 in Z, Fix S2 in X, Fix S5 } \\
\text { in Y, Move S3 in X } t\end{array}$ & $\begin{array}{l}\text { Step 5: Stretch X } \\
\text { Step 6: Release Stretch X }\end{array}$ \\
\hline \hline Shear XY & & $\begin{array}{l}\text { Fix S1 in Z, Fix S4 in Y, Fix S5 } \\
\text { in X and Y, Move S4 in X } s\end{array}$ & $\begin{array}{l}\text { Step 7: Shear YX } \\
\text { Step 8: Release Shear YX }\end{array}$ \\
\hline Shear XY & & $\begin{array}{l}\text { Fix S1 in Z, Fix S2 in X, Fix S3 } \\
\text { in X and Y, Move S2 in Y s }\end{array}$ & $\begin{array}{l}\text { Step 9: Shear XY } \\
\text { Step 10: Release Shear XY }\end{array}$ \\
\hline \hline
\end{tabular}

circumferential displacements of the top of the cylinder were then fixed, while an axial displacement was applied to obtain a prestretch of 1.2. After fully fixing the top, the cylinder was inflated up to $5 \mathrm{kPa}$.

All cases of material implementations enumerated in section 2.4 are tested

on three types of elements, similarly to the previous sections:

- solid incompressible case: full integration hybrid hexahedral elements, $\mathrm{C} 3 \mathrm{D} 8 \mathrm{H}$,

- solid compressible case: full integration hexahedral elements C3D8,

- shell incompressible case: reduced integration plane stress quadrilateral shell elements S4R.

Note that the solid geometry consist of 8400 elements, three times more than the shell geometry, because it has three elements through the thickness of the wall for the same seed size. 
For all test cases, the total CPU time and number of required increments

\subsection{Tests on single elements}

Six cases corresponding to different implementations of the GOH model were compared using a single element on which a variety of deformations were applied. Three types of element were considered: a solid incompressible, a solid compressible and a membrane incompressible element. Figure 4 gives an overview of the results obtained from Abaqus for the hexahedral element: the stress, displacement and reaction force components on a single node at each step. As expected, the results are very similar and appear to overlap.

Therefore, Figure 5 shows the total relative error (see equation 32 reflecting lation (case BIL) which was considered as ground truth. Notice that for the 2D formulation only a selected set of components could be considered. The maximum and minimum error had an order of magnitude of $10^{-4}$ and $10^{-16}$, respectively. As a general trend the errors of UIL were smaller than UAL, UNL, of magnitude.

Figure 6 gives the number of iterations per simulation step that was needed by Abaqus for the different implementations for each element type. For the solid incompressible element, the number of iterations was equal for all cases. For the solid incompressible and membrane incompressible elements, a difference in number of iterations could be observed for some steps. This difference was one 

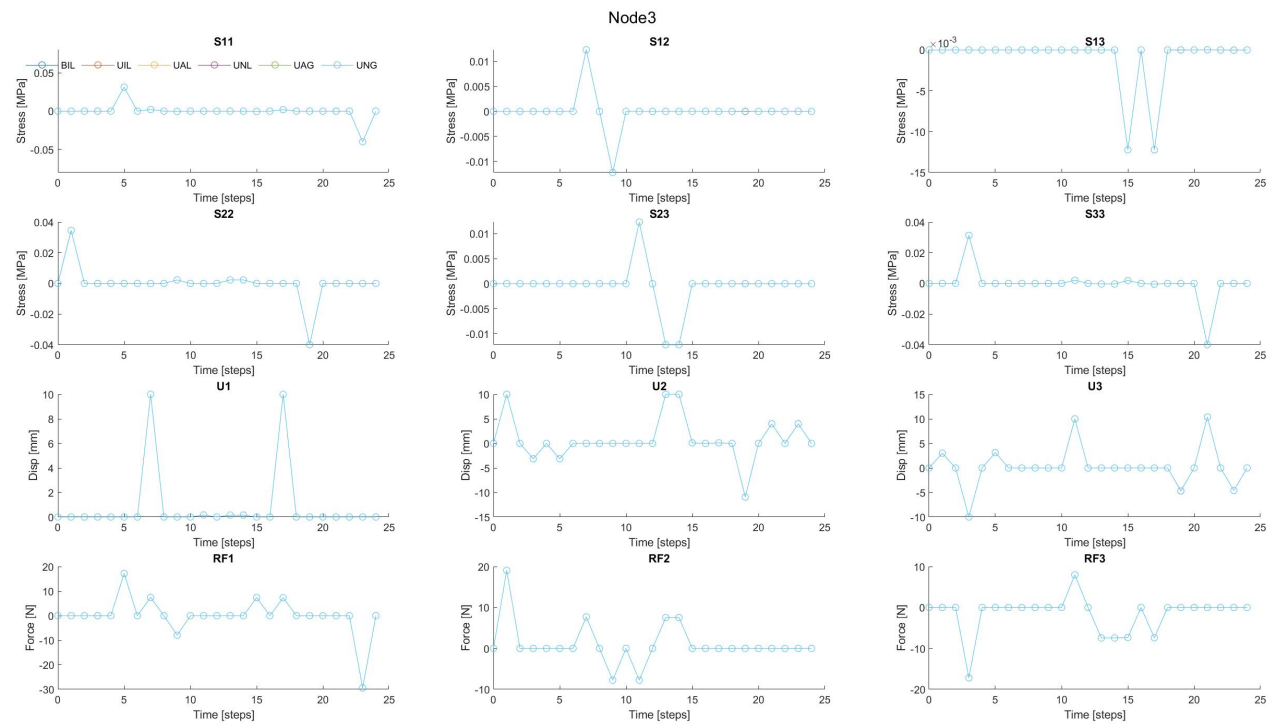

Figure 4: Stress, displacement and reaction force components at node 3 for each step of the solid compressible element. The four different cases (BI, UI, UA and UN) have similar values and appear to overlap. See Figure 5 for the total relative error.

iteration, and the lowest number of iterations was alternating over the different cases.

\subsection{Tests on multiple elements geometry}

Table 6 gives an overview of the results obtained from the extension-inflation test cases explained in section 2.5.1. Note that all simulations with shell elements required an extra increment in the second simulation step. In fact, the start increment size was set to 0.5 to avoid solver issues in the UIL, UAL and UNL cases. In the BIL case this lower increment size is beneficial as well because the CPU time is reduced by avoiding an unsuccessful increment. 

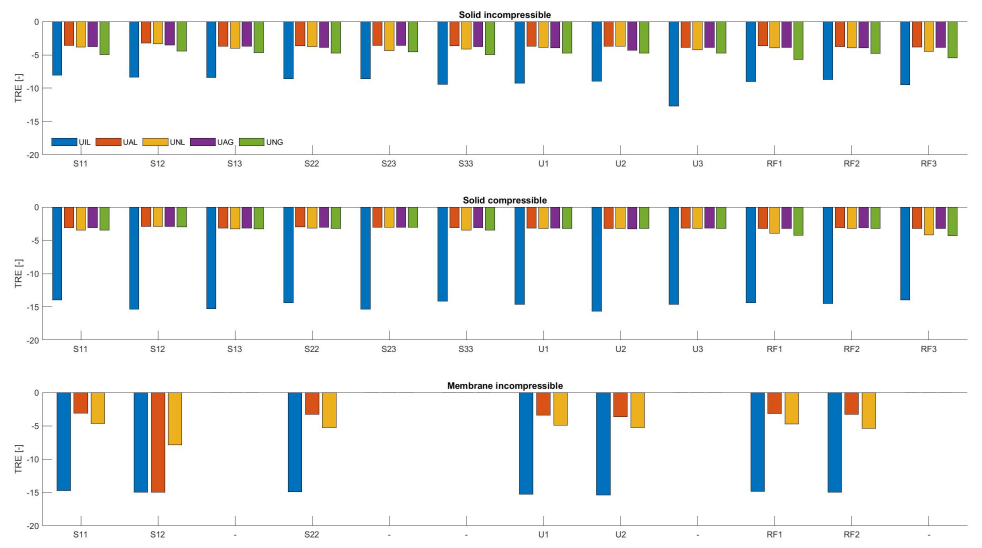

Figure 5: Total relative error on all output components as compared to the results with the built-in material definition. Note that the scale is logarithmic. Note that in the membrane incompressible case, S13, S23, S33, U3 and RF3 are not computed.

\section{Discussion}

\subsection{Comparison of different cases}

The total relative difference between the reference case (BIL) and the other cases was maximally in the order of magnitude of around $10^{-4}$ (see figure 5 and table 6), which can be considered small, taking into account that the difference was summed over the different steps and/or nodes. The trend that the UIL case consistently has the smallest difference of all cases, can be explained by the fact that this implementation only requires the formulation of the derivatives of the invariants, while the stresses and tangent stiffness are calculated by Abaqus. Therefore, this calculation is closest to the calculation of the built-in implementation, against which the difference was calculated. Likewise, the difference of the UAL and UNL cases on the one hand and of the UAG and UNG cases on the other hand are often very similar, which is due to the fact that these implementations use exactly the same stress formulation.

One could argue whether it makes sense to consider the built-in implementation of Abaqus as the reference case, since the exact formulation of the stress 

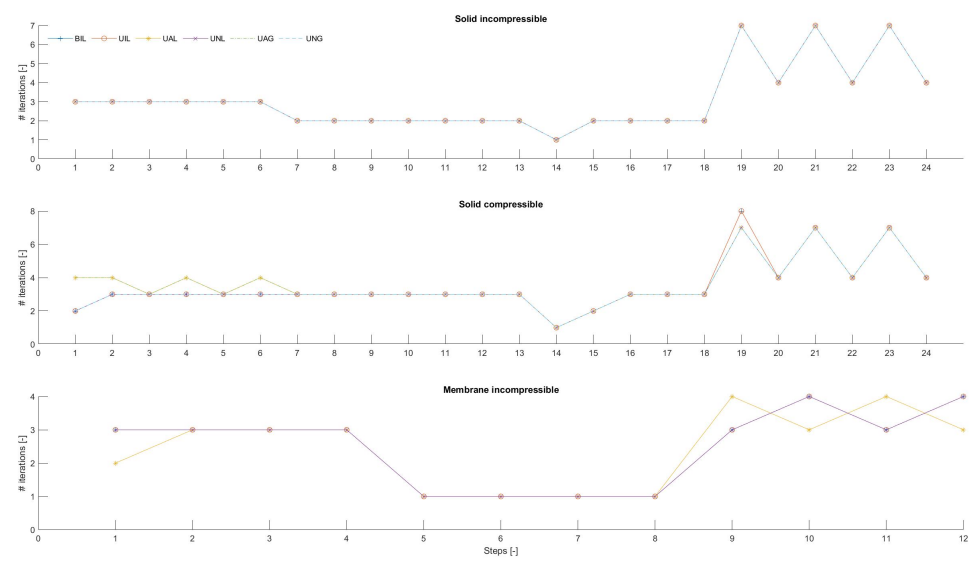

Figure 6: The number of required iterations per simulation step for each case

and stiffness is not available, making it a black box. Changing the reference case (e.g. to UAG), leads to quantitatively similar results regarding the order of magnitude of the maximum error. Regardless of the reference case, the obimplementations to be successful and consider our subroutines accurate enough for most applications within and outside of the field of soft tissue biomechanics.

Regarding the number of iterations and CPU time, the performance of the four implementations was similar (see figure 6 and table 6). Little to no differences in efficiency can be observed in the single element test cases. In the multiple elements simulations, the built-in implementation seems consistently quicker, possibly due to more efficient programming. When comparing the simulation with shell elements versus solid elements, the shell approach is considerably faster, as expected due to the reduced number of elements for the same 405 seed size.

The reported tests show an acceptable performance of the UAL implementation used for shell elements. However, as stated in the Abaqus Theory Guide 1.5.3 2], shell or membrane elements require the computation of the tangent stiffness associated with the Green-Naghdi rate. Despite ignoring this required 
Table 6: Total CPU time, total number of increments over the three simulation steps, total number of iterations and total relative error of the maximal principal stresses (MPS) of all test cases on the multiple elements geometry. $(*)$ The perfect match between case UIL and the BIL reference case is only certain up until the 12 known significant numbers.

\begin{tabular}{lcccc} 
Case & CPU time $[\mathrm{s}]$ & Increments & Iterations & $T R E_{M P S}$ \\
\hline solid incompressible & & & & \\
BIL & 25.4 & 3 & 11 & $-\infty$ \\
UIL & 44.0 & 3 & 11 & -7.56 \\
UAL & 30.2 & 3 & 11 & -3.50 \\
UNL & 31.0 & 3 & 11 & -3.50 \\
UAG & 27.7 & 3 & 11 & -4.28 \\
UNG & 28.3 & 3 & 11 & -4.77 \\
solid compressible & & & & \\
BIL & 24.4 & 3 & 12 & $-\infty$ \\
UIL & 44.9 & 3 & 12 & -7.67 \\
UAL & 56.7 & 3 & 25 & -3.21 \\
UNL & 31.4 & 3 & 12 & -3.34 \\
UAG & 51.8 & 3 & 25 & -2.92 \\
UNG & 28.5 & 3 & 12 & -2.97 \\
shell incompressible & & & & \\
BIL & 7.8 & 4 & 20 & $-\infty$ \\
UIL & 11.5 & 4 & 20 & $-\infty\left(^{*}\right)$ \\
UAL & 11.7 & 4 & 19 & -4.39 \\
UNL & 9.0 & 4 & 20 & -4.50
\end{tabular}

410 correction, our results show a similar convergence rate to the built-in implementation, which may indicate that it is neglected there as well. Examples on how this correction has been taken into account before can be found in [11, 8, 12]. 


\subsection{General remarks and outlook}

The GOH model as implemented in Abaqus has been criticized due to con415 troversy regarding the tension-compression switch, as noted by [13, 14, 15, 16]. In this version, the collagen fibers contribute when $\bar{E}_{i}>0$ in equation 1 . It was chosen due to its availability as a built-in material in Abaqus, since this enabled straightforward comparison. Note, however, that the provided explanations and obtained results are a useful guide for implementing various other invariant-based formulations of anisotropic hyperelastic materials, as well as other rules for the tension-compression switch.

The tutorial also made clear that, when using Abaqus (in versions up to at least version 2019), the user should pay close attention the coordinate system that is used and its effect on the input and output variables in the user subroutine. Most notably, it is counter-intuitive that the deformation gradient is expressed differently for solid continuum than for shell or membrane elements in a UMAT. In that respect, when using the *orientation keyword with solid continuum elements, it would be more intuitive if the received deformation gradient were to map between vectors expressed in the initial and the current local coordinate system rather than have them both expressed in the current coordinate system (see Appendix 8.1 and [3]) for more information).

The different implementations require a different level of complexity, with BIL the least complex and UAL the most complex. However, the increasing complexity also corresponds to an increased freedom to define the material model.

435 In this regard, a UMAT can be used to define material models with more than three fiber families, algorithms that account for constituent-specific deposition stretches [17] or for growth and remodelling of arterial tissue [18. The complex calculation of the analytic tangent stiffness can be avoided by using a numeric approximation of the tangent stiffness. Since the tangent stiffness tensor only affects the speed of convergence, a numerical approximation of the tangent stiffness tensor will not affect the accuracy of the result.

Finally, though this tutorial used Abaqus as a platform, the provided deriva- 
tions can be of use when defining material formulations in many other FE software packages. In the open source package FEBio [19] for example, the GOH

445 constitutive model (see equation 1) is not readily available. However, similar formulations exist in the class of (nearly) incompressible, uncoupled materials. User materials can also be implemented in a material plugin. This plugin requires the computation of the Cauchy stress, according to equation 8 and the stiffness in the form of the spacial elasticity tensor, that corresponds to the first term in equation 17.

\section{Conclusion}

The objective of this paper was to provide a detailed description, at the level of the biomechanical engineer, on how to implement a nonlinear, hyperelastic, fiber-reinforced material model using user subroutines in Abaqus. The

455 GOH material model was used as an example, resulting in six different implementations: BIL (Built-In implementation), UIL (Uanisohyper using Invariant formulation), UAL (Umat in Local coordinate system with Analytical tangent stiffness formulation), UNL (Umat in local coordinate system with Numerical tangent stiffness formulation), UAG (Umat in Global coordinate system with

460 Analytical tangent stiffness) and UNG (Umat in Global coordinate system with Numerical tangent stiffness). Numerical verification of the different implementations shows a good agreement with the built-in implementations in Abaqus. Biomechanical engineers can now use this article and its supplementary material as a starting point to implement their own material models at a preferred level

465 of complexity. Though Abaqus was chosen as a platform here, the derivations are equally useful when using other FE software packages.

\section{Acknowledgements}

This work was supported by a grant strategic basic research (SB 1S35316N), a doctoral fellowship (11A6519N) and two postdoctoral fellowships (PDO/012 470 and $12 \mathrm{ZC} 820 \mathrm{~N}$ ) of the Research Foundation-Flanders (FWO) and a KU Leuven 
category 2 research project (C2-ADAPT). The authors would also like to thank Jamal Seyed Mousavi and David Nolan for their interesting discussions on the topic.

\section{Supplementary material}

Supplementary material to this article contains the input files and the user subroutines and can be found on https://bme-soft-tissue.pages.gitlab. kuleuven.be/UMAT/.

\section{Appendices}

\subsection{Coordinate systems}

Consider the three coordinate systems as defined in section 2.1. We can define two rotation matrices to transform vectors and tensors between different coordinate systems (see Figure 7):

- $Q$ : transformation from the global coordinate system XYZ to the initial local coordinate system xyz.

485

- $\boldsymbol{R}$ : transformation from the global coordinate system XYZ to the current local coordinate system x'y'z'.

The deformation gradient is a two-point tensor, i.e. it maps a vector from the initial, undeformed state to the current, deformed state. Depending on which coordinate system is used to express the vector in the initial and current state, we denote the deformation gradient here as $\boldsymbol{F}^{X Y Z \rightarrow X Y Z}, \boldsymbol{F}^{x y z \rightarrow x^{\prime} y^{\prime} z^{\prime}}$ or $\boldsymbol{F}^{x^{\prime} y^{\prime} z^{\prime} \rightarrow x^{\prime} y^{\prime} z^{\prime}}$. To switch between these expressions, we use [20]:

$$
\begin{aligned}
& \boldsymbol{F}^{x y z \rightarrow x^{\prime} y^{\prime} z^{\prime}}=\boldsymbol{R} \boldsymbol{F}^{X Y Z \rightarrow X Y Z} \boldsymbol{Q}^{T}, \\
& \boldsymbol{F}^{x^{\prime} y^{\prime} z^{\prime} \rightarrow x^{\prime} y^{\prime} z^{\prime}}=\boldsymbol{R} \boldsymbol{F}^{X Y Z \rightarrow X Y Z} \boldsymbol{R}^{T}, \\
& \boldsymbol{F}^{x y z \rightarrow x^{\prime} y^{\prime} z^{\prime}}=\boldsymbol{R} \boldsymbol{F}^{X Y Z \rightarrow X Y Z} \boldsymbol{R}^{T} \boldsymbol{R} \boldsymbol{Q}^{T}=\boldsymbol{F}^{x^{\prime} y^{\prime} z^{\prime} \rightarrow x^{\prime} y^{\prime} z^{\prime}} \boldsymbol{R} \boldsymbol{Q}^{T}
\end{aligned}
$$




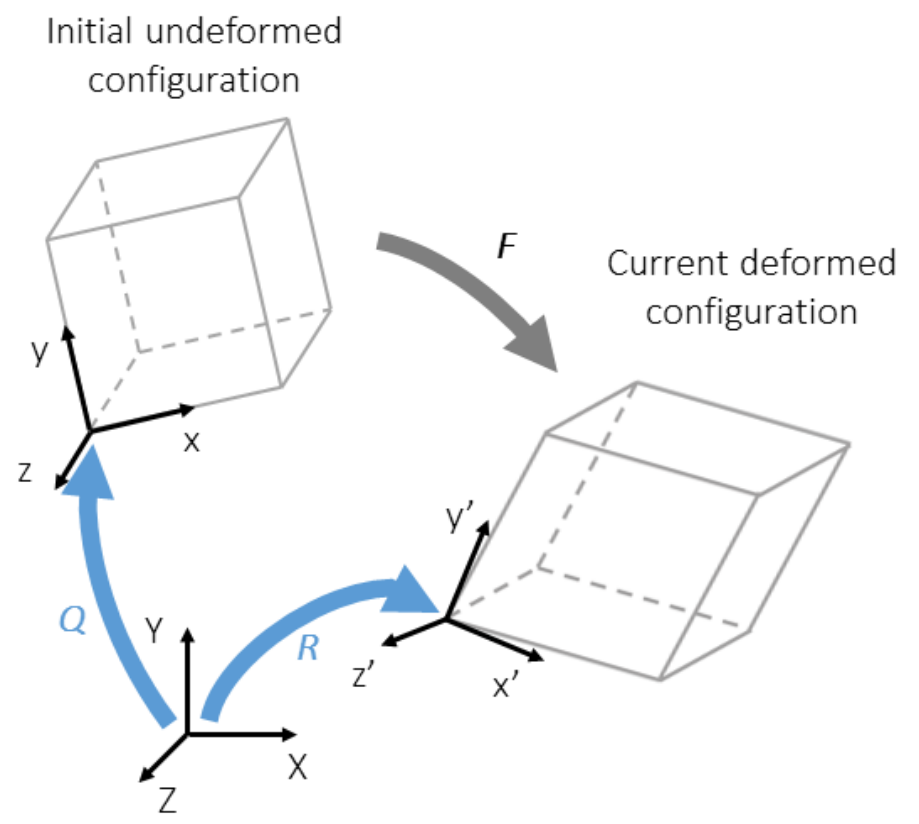

Figure 7: The three considered coordinate systems (the global coordinate system XYZ, the initial local coordinate system xyz, and the current local coordinate system x'y'z'. Two rotation matrices $\boldsymbol{Q}$ and $\boldsymbol{R}$ transform vectors and tensors between the different coordinate systems.

with $\boldsymbol{R}$ and $\boldsymbol{Q}$ as defined before.

When using a local coordinate system, Abaqus needs the stress and Jacobian to be expressed in the current local coordinate system x'y'z'. However, when working with anisotropic, hyperelastic materials, fiber vectors are often unknown in the current local coordinate system x'y'z', but rather known in the initial local coordinate xyz. Hence, it is convenient that Abaqus provides $F^{x y z \rightarrow x^{\prime} y^{\prime} z^{\prime}}$ when using a UMAT with shell or membrane elements (note that a slight deviation from this deformation gradient is possible for shells subjected to out-of-plane deformation, due to bending of the element, see also the Abaqus Theory Guide 3.6.5 [2]). However, for continuum elements, Abaqus provides the deformation gradient in the current local coordinate system $\boldsymbol{F}^{x^{\prime} y^{\prime} z^{\prime} \rightarrow x^{\prime} y^{\prime} z^{\prime}}$. This leaves two 
options to work with anisotropic, hyperelastic materials: It holds that

$$
\boldsymbol{T}^{\prime \prime}=\boldsymbol{Q T} \boldsymbol{Q}^{T},
$$

where $\boldsymbol{T}$ is the rotational component of $\boldsymbol{F}^{X Y Z \rightarrow X Y Z}=\boldsymbol{T} \boldsymbol{U}$. Knowing that $\boldsymbol{T}=\boldsymbol{R}^{T} \boldsymbol{Q}$, one can derive that

$$
\boldsymbol{F}^{x y z \rightarrow x^{\prime} y^{\prime} z^{\prime}}=\boldsymbol{F}^{x^{\prime} y^{\prime} z^{\prime} \rightarrow x^{\prime} y^{\prime} z^{\prime}} \boldsymbol{T}^{\prime \prime T}=\boldsymbol{V}^{\prime \prime} .
$$

In case where the material state is fully determined by the left Cauchy-Green based invariants, this transformation is not required since the difference between $\boldsymbol{F}^{x y z \rightarrow x^{\prime} y^{\prime} z^{\prime}}$ and $\boldsymbol{F}^{x^{\prime} y^{\prime} z^{\prime} \rightarrow x^{\prime} y^{\prime} z^{\prime}}$ cancels out:

$$
\begin{aligned}
\boldsymbol{F}^{x y z \rightarrow x^{\prime} y^{\prime} z^{\prime}} \boldsymbol{F}^{x y z \rightarrow x^{\prime} y^{\prime} z^{\prime T}} & =\boldsymbol{R} \boldsymbol{F}^{X Y Z \rightarrow X Y Z} \boldsymbol{Q}^{T}\left(\boldsymbol{R} \boldsymbol{F}^{X Y Z \rightarrow X Y Z} \boldsymbol{Q}^{T}\right)^{T} \\
& =\boldsymbol{R} F^{X Y Z \rightarrow X Y Z} \boldsymbol{Q}^{T} \boldsymbol{Q} \boldsymbol{F}^{X Y Z \rightarrow X Y Z^{T}} \boldsymbol{R} \\
& =\boldsymbol{R} \boldsymbol{F}^{X Y Z \rightarrow X Y Z} \boldsymbol{F}^{X Y Z \rightarrow X Y Z^{T}} \boldsymbol{R}^{T} \\
& =\boldsymbol{F}^{x y z \rightarrow x^{\prime} y^{\prime} z^{\prime}} \boldsymbol{F}^{x y z \rightarrow x^{\prime} y^{\prime} z^{\prime T}} .
\end{aligned}
$$




\subsection{Expressions for Jacobian matrix}

8.2.1. Relation between equations 14 and 16

Four expressions for the the Jacobian matrix are given in equations $14,15$.

${ }_{525} 16$ and 17 . We show that these are equivalent up to an assumption. Expression 15 can be converted to a formulation in which the partial derivative with respect to the deformation gradient is used by

$$
\begin{aligned}
\mathbb{C} & =\frac{1}{J} \frac{\partial\left(J \boldsymbol{\sigma}-J_{0} \boldsymbol{\sigma}_{0}\right)}{\partial \Delta \boldsymbol{D}}, \\
& =\frac{1}{J} \frac{\partial(J \boldsymbol{\sigma})}{\partial \Delta \boldsymbol{D}}, \\
& =\frac{1}{J} \frac{\partial J\left(\boldsymbol{\sigma}^{i s o}+\boldsymbol{\sigma}^{a n i}\right)}{\partial \Delta \boldsymbol{D}}, \\
& =\frac{1}{J} \frac{\partial\left(J \boldsymbol{\sigma}^{i s o}\right)}{\partial \Delta \boldsymbol{D}}+\frac{1}{J} \frac{\partial\left(J \boldsymbol{\sigma}^{a n i}\right)}{\partial \Delta \boldsymbol{D}} \\
& =\frac{1}{J} \frac{\partial\left(J \boldsymbol{\sigma}^{i s o}\right)}{\partial \boldsymbol{F}}: \frac{\partial \boldsymbol{F}}{\partial \Delta \boldsymbol{D}}+\frac{1}{J} \frac{\partial\left(J \boldsymbol{\sigma}^{a n i}\right)}{\partial \boldsymbol{F}}: \frac{\partial \boldsymbol{F}}{\partial \Delta \boldsymbol{D}} .
\end{aligned}
$$

The rightmost term in the above equation can be found by considering the chain rule:

$$
\frac{\partial \boldsymbol{F}}{\partial \Delta \boldsymbol{D}}=\frac{\partial \boldsymbol{F}}{\partial \Delta \boldsymbol{L}}: \frac{\partial \Delta \boldsymbol{L}}{\partial \Delta \boldsymbol{D}},
$$

530

Abaqus as $\boldsymbol{L} \Delta t$, and which can be approximated as

$$
\Delta \boldsymbol{L}=\Delta \boldsymbol{F} \cdot \hat{\boldsymbol{F}}^{-1}
$$

where $\hat{\boldsymbol{F}}$ is the average deformation gradient over the increment, and $\Delta \boldsymbol{F}$ is the increment of the deformation gradient. Both are given in terms of the deformation gradient at the beginning and the end of the increment as

$$
\begin{aligned}
\hat{\boldsymbol{F}} & =\frac{1}{2}\left(\boldsymbol{F}_{0}+\boldsymbol{F}\right) \\
\Delta \boldsymbol{F} & =\boldsymbol{F}-\boldsymbol{F}_{0} .
\end{aligned}
$$

535 We know that

$$
\Delta \boldsymbol{L}=\Delta \boldsymbol{D}+\Delta \boldsymbol{W}
$$


whereby the first term is symmetric and the second term is asymmetric. Since $\Delta \boldsymbol{D}$ is symmetric, we can write

$$
\begin{aligned}
\Delta D_{i j} & =\operatorname{sym}(\boldsymbol{\Delta} \boldsymbol{D})_{i j} \\
& =\frac{1}{2}\left(\Delta D_{i j}+\Delta D_{j i}\right) \\
& =\frac{1}{2}\left(\delta_{i a} \delta_{j b}+\delta_{i b} \delta_{j a}\right) \Delta D_{a b} .
\end{aligned}
$$

Hence,

$$
\begin{aligned}
\left(\frac{\partial \Delta \boldsymbol{W}}{\partial \Delta \boldsymbol{D}}\right)_{i j k l} & =0 \\
\left(\frac{\partial \Delta \boldsymbol{D}}{\partial \Delta \boldsymbol{D}}\right)_{i j k l} & =\frac{1}{2}\left(\delta_{i a} \delta_{j b}+\delta_{i b} \delta_{j a}\right) \delta_{a k} \delta_{b l} \\
& =\frac{1}{2}\left(\delta_{i k} \delta_{j l}+\delta_{i l} \delta_{j k}\right) \\
\left(\frac{\partial \Delta \boldsymbol{L}}{\partial \Delta \boldsymbol{D}}\right)_{i j k l} & =\frac{1}{2}\left(\delta_{i k} \delta_{j l}+\delta_{i l} \delta_{j k}\right) .
\end{aligned}
$$

We can write

$$
\begin{aligned}
\frac{\partial \Delta F_{i j}}{\partial F_{k l}} & =\delta_{i k} \delta_{j l} \\
\frac{\partial \hat{F}_{i j}}{\partial F_{k l}} & =\frac{1}{2} \delta_{i k} \delta_{j l},
\end{aligned}
$$


such that

$$
\begin{aligned}
\left(\frac{\partial \boldsymbol{\Delta} \boldsymbol{L}}{\partial \boldsymbol{F}}\right)_{i j k l} & =\frac{\partial\left(\boldsymbol{\Delta} \boldsymbol{F} \cdot \hat{\boldsymbol{F}}^{-1}\right)_{i j}}{\partial F_{k l}} \\
& =\frac{\partial \Delta F_{i a}}{\partial F_{k l}}\left(\hat{\boldsymbol{F}}^{-1}\right)_{a j}+\Delta F_{i a} \frac{\partial\left(\hat{\boldsymbol{F}}^{-1}\right)_{a j}}{\partial F_{k l}} \\
& =\frac{\partial \Delta F_{i a}}{\partial F_{k l}}\left(\hat{\boldsymbol{F}}^{-1}\right)_{a j}+\Delta F_{i a}\left(-\left(\hat{\boldsymbol{F}}^{-1}\right)_{a b} \frac{\partial \hat{F}_{b c}}{\partial F_{k l}}\left(\hat{\boldsymbol{F}}^{-1}\right)_{c j}\right) \\
& =\delta_{i k} \delta_{a l}\left(\hat{\boldsymbol{F}}^{-1}\right)_{a j}-\frac{1}{2} \Delta F_{i a}\left(\hat{\boldsymbol{F}}^{-1}\right)_{a b} \delta_{b k} \delta_{c l}\left(\hat{\boldsymbol{F}}^{-1}\right)_{c j} \\
& =\delta_{i k}\left(\hat{\boldsymbol{F}}^{-1}\right)_{l j}-\frac{1}{2}\left(\Delta \boldsymbol{F} \hat{\boldsymbol{F}}^{-1}\right)_{i k}\left(\hat{\boldsymbol{F}}^{-1}\right)_{l j} \\
& =\left[\left(\hat{\boldsymbol{F}} \hat{\boldsymbol{F}}^{-1}\right)_{i k}-\frac{1}{2}\left(\Delta \boldsymbol{F} \hat{\boldsymbol{F}}^{-1}\right)_{i k}\right]\left(\hat{\boldsymbol{F}}^{-1}\right)_{l j} \\
& =\left(\hat{F}_{i a}-\frac{1}{2} \Delta F_{i a}\right)\left(\hat{\boldsymbol{F}}^{-1}\right)_{a k}\left(\hat{\boldsymbol{F}}^{-1}\right)_{l j} \\
& =F_{0 i a}\left(\hat{\boldsymbol{F}}^{-1}\right)_{a k}\left(\hat{\boldsymbol{F}}^{-1}\right)_{l j} \\
& =\left(\boldsymbol{F}_{0} \cdot \hat{\boldsymbol{F}}^{-1}\right)_{i k}\left(\hat{\boldsymbol{F}}^{-1}\right)_{l j} .
\end{aligned}
$$

It can be shown that

$$
f(\boldsymbol{F})=\left(\boldsymbol{F}-\boldsymbol{F}_{0}\right) \cdot\left(\frac{1}{2}\left(\boldsymbol{F}+\boldsymbol{F}_{0}\right)\right)^{-1}=\Delta \boldsymbol{L}
$$

is an invertible function. Therefore,

$$
\left(\frac{\partial \boldsymbol{F}}{\partial \Delta \boldsymbol{L}}\right)_{i j k l}=\left(\hat{\boldsymbol{F}} \cdot \boldsymbol{F}_{0}^{-1}\right)_{i k}(\hat{\boldsymbol{F}})_{l j} .
$$

Bringing equations 47 and 51 together, we get

$$
\begin{aligned}
\left(\frac{\partial \boldsymbol{F}}{\partial \Delta \boldsymbol{D}}\right)_{i j k l} & =\left(\frac{\partial \boldsymbol{F}}{\partial \Delta \boldsymbol{L}}\right)_{i j a b}\left(\frac{\partial \Delta \boldsymbol{L}}{\partial \Delta \boldsymbol{D}}\right)_{a b k l} \\
& =\frac{1}{2}\left(\hat{\boldsymbol{F}} \boldsymbol{F}_{0}^{-1}\right)_{i b} \hat{F}_{b j}\left(\delta_{a k} \delta_{b l}+\delta_{a l} \delta_{b k}\right) \\
& =\frac{1}{2}\left[\left(\hat{\boldsymbol{F}} \boldsymbol{F}_{0}^{-1}\right)_{i k} \hat{F}_{l j}+\left(\hat{\boldsymbol{F}} \boldsymbol{F}_{0}^{-1}\right)_{i l} \hat{F}_{k j}\right] .
\end{aligned}
$$

The remaining terms in equation 41 can be obtained by differentiating the ${ }_{545}$ Cauchy stresses of the considered constitutive model with respect to the deformation gradient. 
It is possible to obtain a more concise formulation of the Jacobian matrix, in accordance to literature [6] as shown in equation 16. Making the assumption that $\boldsymbol{F}_{0}=\boldsymbol{F}$, then $\hat{\boldsymbol{F}}=\boldsymbol{F}$, we can write

$$
\left(\frac{\partial \boldsymbol{F}}{\partial \Delta \boldsymbol{D}}\right)_{i j k l}=\frac{1}{2}\left(\delta_{i k} F_{l j}+\delta_{i l} F_{k j}\right) .
$$

In index notation, equation 41 becomes

$$
\begin{aligned}
\mathbb{C}_{i j k l} & =\frac{1}{J} \frac{\partial\left(J \sigma_{i j}\right)}{\partial F_{a b}} \frac{\partial F_{a b}}{\partial \Delta D_{k l}} \\
& =\frac{1}{J}\left(J \frac{\partial \sigma_{i j}}{\partial F_{a b}}+\frac{\partial J}{\partial F_{a b}} \sigma_{i j}\right) \frac{\partial F_{a b}}{\partial \Delta D_{k l}} \\
& =\left(\frac{\partial \sigma_{i j}}{\partial F_{a b}}+\left(\boldsymbol{F}^{-T}\right)_{a b} \sigma_{i j}\right) \frac{\partial F_{a b}}{\partial \Delta D_{k l}} \\
& =\frac{\partial \sigma_{i j}}{\partial F_{a b}} \frac{\partial F_{a b}}{\partial \Delta D_{k l}}+\left(\boldsymbol{F}^{-T}\right)_{a b} \sigma_{i j} \frac{\partial F_{a b}}{\partial \Delta D_{k l}}
\end{aligned}
$$

Considering the first term,

$$
\begin{aligned}
\frac{\partial \sigma_{i j}}{\partial F_{a b}} \frac{\partial F_{a b}}{\partial \Delta D_{k l}} & =\frac{\partial \sigma_{i j}}{\partial F_{a b}} \frac{1}{2}\left(\delta_{a k} F_{l b}+\delta_{a l} F_{k b}\right) \\
& =\frac{1}{2}\left(\frac{\partial \sigma_{i j}}{\partial F_{k b}} F_{l b}+\frac{\partial \sigma_{i j}}{\partial F_{l b}} F_{k b}\right) .
\end{aligned}
$$

The second term becomes

$$
\begin{aligned}
\left(\boldsymbol{F}^{-T}\right)_{a b} \sigma_{i j} \frac{\partial F_{a b}}{\partial \Delta D_{k l}} & =\left(\boldsymbol{F}^{-T}\right)_{a b} \sigma_{i j} \frac{1}{2}\left(\delta_{a k} F_{l b}+\delta_{a l} F_{k b}\right) \\
& =\frac{1}{2} \sigma_{i j}\left(\left(\boldsymbol{F}^{-T}\right)_{k b} F_{l b}+\left(\boldsymbol{F}^{-T}\right)_{l b} F_{k b}\right) \\
& =\frac{1}{2} \sigma_{i j}\left(\delta_{k l} \delta_{l k}\right) \\
& =\sigma_{i j} \delta_{k l} .
\end{aligned}
$$

Adding the results of equations 55 and 56 corresponds exactly to equation 16 This concise formulation may therefore be used to derived the Jacobian tensor in 555 a UMAT, keeping in mind that one approximation is made. Namely, it is assumed that $\boldsymbol{F}_{0}=\boldsymbol{F}$. 


\subsubsection{Relation between equations 16 and 17}

Equation 17 can be derived from equation 16 to show the equivalence. First, it can be written that

$$
\begin{aligned}
\frac{\partial \sigma_{i j}}{\partial F_{k a}} & =\frac{\partial\left(\frac{1}{J} F_{i c} S_{c b} F_{j b}\right)}{\partial F_{k a}} \\
& =\frac{\partial\left(\frac{1}{J}\right)}{\partial F_{k a}} F_{i c} S_{c b} F_{j b}+\frac{1}{J} \frac{\partial F_{i c}}{\partial F_{k a}} S_{c b} F_{j b}+\frac{1}{J} F_{i c} \frac{\partial S_{c b}}{\partial S_{k a}} F_{j b}+\frac{1}{J} F_{i c} S_{c b} \frac{\partial F_{j b}}{\partial F_{k a}} \\
& =-\frac{1}{J}\left(\boldsymbol{F}^{-T}\right)_{k a} F_{i c} S_{c b} F_{j b}+\frac{1}{J} \delta_{i k} \delta_{c a} S_{c b} F_{j b}+\frac{1}{J} F_{i c} \frac{\partial S_{c b}}{\partial F_{k a}} F_{j b}+\frac{1}{J} F_{i c} S_{c b} \delta_{j k} \delta_{b a} \\
& =\frac{1}{J}\left(-\left(\boldsymbol{F}^{-T}\right)_{k a} F_{i c} S_{c b} F_{j b}+\delta_{i k} S_{a b} F_{j b}+F_{i c} \frac{\partial S_{c b}}{\partial F_{k a}} F_{j b}+F_{i c} S_{c a} \delta_{j k}\right) .
\end{aligned}
$$

560

Therefore,

$$
\begin{aligned}
\mathbb{C}_{i j k l}= & \sigma_{i j} \delta_{k l}+\frac{1}{2}\left(\frac{\partial \sigma_{i j}}{\partial F_{k a}} F_{l a}+\frac{\partial \sigma_{i j}}{\partial F_{l a}} F_{k a}\right) \\
= & \sigma_{i j} \delta_{k l}+\frac{1}{2 J}\left[-\left(\boldsymbol{F}^{-T}\right)_{k a} F_{i c} \delta_{c b} F_{j b} F_{l a}-\left(\boldsymbol{F}^{-T}\right)_{l a} F_{i c} \delta_{c b} F_{j b} F_{k a}\right. \\
& +\delta_{i k} S_{a b} F_{j b} F_{l a}+\delta_{i l} S_{a b} F_{j b} F_{k a}+F_{i c} \frac{\partial S_{c b}}{\partial F_{k a}} F_{j b} F_{l a}+F_{i c} \frac{\partial S_{c b}}{\partial F_{l a}} F_{j b} F_{k a} \\
& \left.+F_{i c} \delta_{c a} \delta_{j k} F_{l a}+F_{i c} S_{c a} \delta_{j l} F_{k a}\right] \\
= & \frac{1}{J}\left(\boldsymbol{F} \boldsymbol{S} \boldsymbol{F}^{T}\right)_{i j} \delta_{k l}+\frac{1}{2 J}\left[-\delta_{k l}\left(\boldsymbol{F} \boldsymbol{S} \boldsymbol{F}^{T}\right)_{i j}-\delta_{l k}\left(\boldsymbol{F} \boldsymbol{S} \boldsymbol{F}^{T}\right)_{i j}\right. \\
& +\delta_{i k}\left(\boldsymbol{F} \boldsymbol{S} \boldsymbol{F}^{T}\right)_{l j}+\delta_{i l}\left(\boldsymbol{F} \boldsymbol{S} \boldsymbol{F}^{T}\right)_{k j}+F_{i c} \frac{\partial S_{c b}}{\partial F_{k a}} F_{j b} F_{l a}+F_{i c} \frac{\partial S_{c b}}{\partial F_{l a}} F_{j b} F_{k a} \\
& \left.+\delta_{j k}\left(\boldsymbol{F} \boldsymbol{S} \boldsymbol{F}^{T}\right)_{i l}+\delta_{j l}\left(\boldsymbol{F} \boldsymbol{S} \boldsymbol{F}^{T}\right)_{i k}\right] \\
= & \frac{1}{2}\left(\delta_{i k} \sigma_{l j}+\delta_{i l} \sigma_{k j}+\delta_{j k} \sigma_{i l}+\delta_{j l} \sigma_{i k}\right)+\frac{1}{J} F_{i c} \frac{\partial S_{c b}}{\partial C_{a d}} F_{k d} F_{j b} F_{l a}+\frac{1}{J} F_{i c} \frac{\partial S_{c b}}{\partial C_{a d}} F_{l d} F_{j b} F_{k a}
\end{aligned}
$$

from $\frac{\partial S_{i j}}{\partial F_{k a}}=2 \frac{\partial S_{i j}}{\partial C_{a b}} F_{k b}$ (see also equation 72. Knowing that $\boldsymbol{\sigma}$ and $\boldsymbol{C}$ are symmetric tensors, this is equivalent to equation 17. Therefore, equations 16 and 17 refer to the exact same tensor.

\subsubsection{Relation between equations 15 and 17}

Equation 17 also follows directly from equation 15 . The derivation to follow is largely inspired by [21]. Knowing from the Abaqus Subroutine Reference 
Guide 1.1.41 [2] that

$$
\begin{gathered}
\delta \boldsymbol{D}=\operatorname{sym}\left(\delta \boldsymbol{F} \cdot \boldsymbol{F}^{-1}\right) \\
\delta \boldsymbol{W}=\operatorname{asym}\left(\delta \boldsymbol{F} \cdot \boldsymbol{F}^{-1}\right),
\end{gathered}
$$

we can write

$$
\begin{gathered}
\delta \boldsymbol{D}=\operatorname{sym}\left(\dot{\boldsymbol{F}} \cdot \boldsymbol{F}^{-1}\right)=\operatorname{sym}(\boldsymbol{L})=\boldsymbol{D} \\
\delta \boldsymbol{W}=\operatorname{asym}\left(\dot{\boldsymbol{F}} \cdot \boldsymbol{F}^{-1}\right)=\operatorname{asym}(\boldsymbol{L})=\boldsymbol{W},
\end{gathered}
$$

where the variation of the deformation gradient $\delta \boldsymbol{F}$ is approximated by its time derivative $\dot{\boldsymbol{F}}$. Equation 15 is then rewritten to

$$
\dot{\tau}=J \mathbb{C}: \boldsymbol{D}+\boldsymbol{W} \cdot \boldsymbol{\tau}-\boldsymbol{\tau} \cdot \boldsymbol{W}
$$

where $\boldsymbol{\tau}=J \boldsymbol{\sigma}$ is the Kirchhoff stress tensor. It can be shown that the ZarembaJaumann rate of the Kirchhoff stress may be written as

$$
\stackrel{\Delta}{\tau}=\dot{\tau}-W \cdot \tau+\tau \cdot W
$$

This is also known as the Lie-derivative 22 Combining equations 61 and 62 gives

$$
\stackrel{\Delta}{\tau}=J \mathbb{C}: \boldsymbol{D}
$$

575 such that we are looking for a stiffness tensor $\mathbb{C}$ related to the Zaremba-Jaumann stress rate. The Truesdell rate of the Kirchhoff stress may be written as

$$
\stackrel{o}{\boldsymbol{\tau}}=\dot{\boldsymbol{\tau}}-\boldsymbol{L} \cdot \boldsymbol{\tau}-\boldsymbol{\tau} \cdot \boldsymbol{L}^{T}
$$

Knowing that $\boldsymbol{L}=\boldsymbol{W}+\boldsymbol{D}$ and that $\boldsymbol{D}$ is a symmetric tensor and $\boldsymbol{W}$ an antisymmetric tensor,

$$
\stackrel{o}{\boldsymbol{\tau}}=\stackrel{\Delta}{\boldsymbol{\tau}}-\boldsymbol{D} \cdot \boldsymbol{\tau}-\boldsymbol{\tau} \cdot \boldsymbol{D}
$$

such that

$$
\begin{aligned}
J \mathbb{C}: \boldsymbol{D} & =\stackrel{o}{\boldsymbol{\tau}}+\boldsymbol{D} \cdot \boldsymbol{\tau}+\boldsymbol{\tau} \cdot \boldsymbol{D} \\
\Rightarrow \mathbb{C}: \boldsymbol{D} & =\boldsymbol{c}: \boldsymbol{D}+\boldsymbol{D} \cdot \boldsymbol{\sigma}+\boldsymbol{\sigma} \cdot \boldsymbol{D},
\end{aligned}
$$

580

where $\stackrel{o}{\boldsymbol{\tau}}=J \boldsymbol{c}: \boldsymbol{D}$ and $\boldsymbol{c}$ is the stiffness tensor associated with the Truesdell rate of the Kirchhoff stress. $\mathbb{C}$ can divided in two parts as $\boldsymbol{c}+\boldsymbol{c}^{\prime}$, with

$$
c^{\prime}: D=D \cdot \sigma+\sigma \cdot D
$$


Many different tensors $\boldsymbol{c}^{\prime}$ satisfy this equation. Adding the requirements of major and minor symmetries, i.e. $c_{i j k l}^{\prime}=c_{l k j i}^{\prime}$ and $c_{i j k l}^{\prime}=c_{j i k l}^{\prime}=c_{i j l k}^{\prime}=c_{j i l k}^{\prime}$, we obtain

$$
c_{i j k l}^{\prime}=\frac{1}{2}\left(\delta_{i k} \sigma_{j l}+\delta_{i l} \sigma_{j k}+\delta_{j k} \sigma_{i l}+\delta_{j l} \sigma_{i k}\right) .
$$

To obtain the tensor $\boldsymbol{c}$, we use the definition of $\stackrel{o}{\boldsymbol{\tau}}$ :

$$
\stackrel{o}{\boldsymbol{\tau}}=\boldsymbol{F} \cdot \dot{\boldsymbol{S}} \cdot \boldsymbol{F}^{T}
$$

and the following equality:

$$
\begin{aligned}
\boldsymbol{F}^{-T} \cdot \dot{\boldsymbol{E}} \cdot \boldsymbol{F}^{-1} & =\boldsymbol{F}^{-T} \cdot \frac{\partial}{\partial t}\left(\frac{1}{2}\left(\boldsymbol{F}^{T} \boldsymbol{F}-\boldsymbol{I}\right)\right) \cdot \boldsymbol{F}^{-1} \\
& =\frac{1}{2} \boldsymbol{F}^{-T}\left(\boldsymbol{F}^{T} \dot{\boldsymbol{F}}+\dot{\boldsymbol{F}}^{T} \boldsymbol{F}\right) \boldsymbol{F}^{-1} \\
& =\frac{1}{2}\left(\dot{\boldsymbol{F}} \boldsymbol{F}^{-1}+\boldsymbol{F}^{-T} \dot{\boldsymbol{F}}^{T}\right) \\
& =\frac{1}{2}\left(\boldsymbol{L}+\boldsymbol{L}^{T}\right) \\
& =\boldsymbol{D}
\end{aligned}
$$

where $\dot{\boldsymbol{E}}$ is the time derivative of the Green-Lagrange strain tensor and $\dot{\boldsymbol{S}}$ the time derivative of the second Piola-Kirchhoff stress. Earlier, we wrote $\stackrel{o}{\boldsymbol{\tau}}=J \boldsymbol{c}$ : $\boldsymbol{D}$, such that we can find $\boldsymbol{c}$ as

$$
\begin{aligned}
J c_{i j k l} D_{k l} & =\stackrel{o}{\tau}_{i j} \\
\Rightarrow J c_{i j k l} F_{c k}^{-1} \dot{E}_{c d} F_{d l}^{-1} & =F_{i a} \dot{S}_{a b} F_{j b} \\
\Rightarrow c_{i j k l} & =\frac{1}{J} \frac{\dot{S}_{a b}}{\dot{E}_{c d}} F_{i a} F_{j b} F_{k c} F_{l d} \\
& =\frac{1}{J}\left(\frac{\partial \boldsymbol{S}}{\partial \boldsymbol{E}}\right)_{a b c d} F_{i a} F_{j b} F_{k c} F_{l d} \\
& =\frac{2}{J}\left(\frac{\partial \boldsymbol{S}}{\partial \boldsymbol{C}}\right)_{a b c d} F_{i a} F_{j b} F_{k c} F_{l d} \\
& =\frac{4}{J}\left(\frac{\partial^{2} \Psi}{(\partial \boldsymbol{C})^{2}}\right)_{a b c d} F_{i a} F_{j b} F_{k c} F_{l d} .
\end{aligned}
$$

590 Combining equations 68 and 71 , gives us the final expression for $\mathbb{C}$ we were looking for. 


\subsubsection{Relation between equations 14 and 15}

Finally, to relate the two equations for the Jacobian, given in the Abaqus manual (User Subroutines Reference Guide 1.1.41 [2]), the following observa-

595 latter by $\delta \boldsymbol{D}$, taking into account that $\boldsymbol{D}$ and $\boldsymbol{W}$ are independent and setting $\delta \boldsymbol{D}=\Delta \epsilon$ as done earlier. Formally, this is not a division, because the variation in Kirchhoff stress depends on both the variation in rate of deformation $\boldsymbol{D}$ and the rate of rotation $\boldsymbol{W}$. However, in practice, Abaqus is only interested in the variation of Kirchhoff stress with respect to the rate of deformation, such that the rotational terms are discarded.

\subsection{Other expressions}

Knowing that $C$ is a symmetric tensor, we can write

$$
\begin{aligned}
\frac{\partial \Psi}{\partial \boldsymbol{F}} & =\frac{\partial \Psi}{\partial C}: \frac{\partial \boldsymbol{C}}{\partial \boldsymbol{F}} \\
\Rightarrow\left(\frac{\partial \Psi}{\partial \boldsymbol{F}}\right)_{k l} & =\left(\frac{\partial \Psi}{\partial \boldsymbol{C}}\right)_{i j}\left(\frac{\partial \boldsymbol{C}}{\partial \boldsymbol{F}}\right)_{i j k l} \\
& =\left(\frac{\partial \Psi}{\partial \boldsymbol{C}}\right)_{i j}\left(\delta_{i l} F_{k j}+F_{k i} \delta_{j l}\right) \\
& =\left(\frac{\partial \Psi}{\partial \boldsymbol{C}}\right)_{l j} F_{k j}+\left(\frac{\partial \Psi}{\partial \boldsymbol{C}}\right)_{i l} F_{k i} \\
& =\left(\frac{\partial \Psi}{\partial \boldsymbol{C}} \boldsymbol{F}^{T}\right)_{l k}+\left(\boldsymbol{F} \frac{\partial \Psi}{\partial \boldsymbol{C}}\right)_{k l} \\
& =2\left(\boldsymbol{F} \frac{\partial \Psi}{\partial \boldsymbol{C}}\right)_{k l} .
\end{aligned}
$$

\section{References} arterial layers with distributed collagen fibre orientations (2006). arXiv: 0312002v1, doi:10.1098/rsif.2005.0073.

[2] Abaqus 6.14 Documentation. 
[3] D. R. Nolan, C. Lally, J. P. McGarry, Understanding the deformation gradi-

[ [8] H. Liu, W. Sun, Numerical Approximation of Elasticity Tensor Associated With Green-Naghdi Rate, Journal of Biomechanical Engineering 139 (8) (2017) 81007. doi:10.1115/1.4036829.

11 URL http://biomechanical.asmedigitalcollection.asme.org/ article.aspx?doi=10.1115/1.4036829

9] W. Sun, E. L. Chaikof, M. E. Levenston, Numerical Approximation of Tangent Moduli for Finite Element Implementations of Nonlinear Hyperelastic Material Models, Journal of Biomechanical Engineering 130 (6) (2008) 61003. doi:10.1115/1.2979872. subroutines (UMATs), Journal of the Mechanical Behavior of Biomedical Materials (submitted).

[4] J. Bonet, R. D. Wood, Nonlinear Continuum Mechanics for Finite Element Analysis, Cambridge University Press, 1997.

5] G. A. Holzapfel, Nonlinear Solid Mechanics: A Continuum Approach for Engineering, John Wiley \& Sons, 2000.

[6] D. R. Nolan, A. L. Gower, M. Destrade, R. W. Ogden, J. P. McGarry, A robust anisotropic hyperelastic formulation for the modelling of soft tissue, Journal of the Mechanical Behavior of Biomedical Materials 39 (2014) 4860. doi:10.1016/j.jmbbm.2014.06.016.

[7] H. Liu, W. Sun, Computational efficiency of numerical approximations of tangent moduli for finite element implementation of a fiberreinforced hyperelastic material model, Computer Methods in Biomechanics and Biomedical Engineering 19 (11) (2016) 1171-1180. doi:10.1080/ 10255842.2015 .1118467 .

URL http://biomechanical.asmedigitalcollection.asme.org/ article.aspx?articleid $=1475570$ 
[10] J. Vastmans, H. Fehervary, P. Verbrugghe, T. Verbelen, E. Vanderveken, J. Vander Sloten, T. Treasure, F. Rega, N. Famaey, Biomechanical evaluation of a personalized external aortic root support applied in the Ross procedure, Journal of the Mechanical Behavior of Biomedical Materialsdoi:10.1016/j.jmbbm.2017.11.018

[11] V. Prot, B. Skallerud, G. A. Holzapfel, Transversely isotropic membrane shells with application to mitral valve mechanics. Constitutive modelling and finite element implementation, International Journal for Numerical Methods in Engineering 71 (8) (2007) 987-1008. arXiv:1010.1724, doi: $10.1002 / \mathrm{nme} .1983$.

[12] C. Bellini, S. Federico, Green-Naghdi rate of the Kirchhoff stress and deformation rate: the elasticity tensor, Zeitschrift fur Angewandte Mathematik und Physikdoi:10.1007/s00033-014-0421-x.

${ }_{650}$ [13] G. A. Holzapfel, R. W. Ogden, On the tension-compression switch in soft 1 f fibrous solids, European Journal of Mechanics, A/Solidsdoi:10.1016/j. euromechsol.2014.09.005.

[14] A. V. Melnik, H. Borja Da Rocha, A. Goriely, On the modeling of fiber dispersion in fiber-reinforced elastic materials, International Journal of NonLinear Mechanicsdoi:10.1016/j.ijnonlinmec.2014.10.006.

[15] M. Latorre, F. J. Montáns, On the tension-compression switch of the Gasser-Ogden-Holzapfel model: Analysis and a new pre-integrated proposal, Journal of the Mechanical Behavior of Biomedical Materialsdoi: 10.1016/j.jmbbm.2015.11.018.

[16] G. A. Holzapfel, R. W. Ogden, S. Sherifova, On fibre dispersion modelling of soft biological tissues: A review (2019). doi:10.1098/rspa.2018.0736.

[17] L. Maes, H. Fehervary, J. Vastmans, S. J. Mousavi, S. Avril, N. Famaey, Constrained mixture modeling affects material parameter identification 
from planar biaxial tests, Journal of the Mechanical Behavior of Biomedical Materials 95 (2019) 124-135. doi:10.1016/j.jmbbm.2019.03.029.

[18] N. Famaey, J. Vastmans, H. Fehervary, L. Maes, E. Vanderveken, F. Rega, S. J. Mousavi, S. Avril, Numerical simulation of arterial remodeling in pulmonary autografts, ZAMM - Journal of Applied Mathematics and Mechanics / Zeitschrift für Angewandte Mathematik und Mechanikdoi: 10.1002/zamm.201700351.

[19] FEBio Software Suite, Finite Elements for Biomechanics.

[20] I.-S. Liu, On the Transformation Property of the Deformation Gradient under a Change of Frame, Journal of Elasticity 71 (1-3) (2003) 73-80. doi:10.1023/b:elas.0000005548.36767.e7.

675 [21] M. Holland, The Hitchhiker's Guide to Abaqusdoi:10.5281/zenodo. 1243269

[22] T. Belytschko, W. K. Liu, B. Moran, Nonlinear finite elements for continua and structures, John Wiley and Sons Ltd, 2000. 